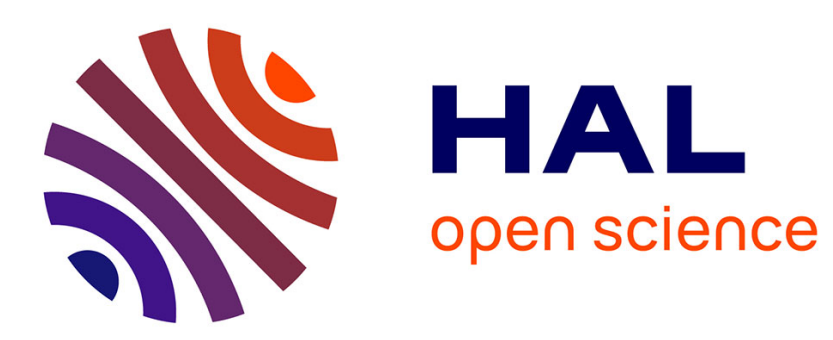

\title{
Fokker-Plank System for Movement of Micro-organism Population in Confined Environment
}

\author{
Jingyi Fu, Benoit Perthame, Min Tang
}

\section{To cite this version:}

Jingyi Fu, Benoit Perthame, Min Tang. Fokker-Plank System for Movement of Micro-organism Population in Confined Environment. Journal of Statistical Physics, 2021, 184 (1), 10.1007/s10955-02102760-y . hal-03278860

\section{HAL Id: hal-03278860 \\ https://hal.sorbonne-universite.fr/hal-03278860}

Submitted on 6 Jul 2021

HAL is a multi-disciplinary open access archive for the deposit and dissemination of scientific research documents, whether they are published or not. The documents may come from teaching and research institutions in France or abroad, or from public or private research centers.
L'archive ouverte pluridisciplinaire HAL, est destinée au dépôt et à la diffusion de documents scientifiques de niveau recherche, publiés ou non, émanant des établissements d'enseignement et de recherche français ou étrangers, des laboratoires publics ou privés. 


\title{
Fokker-Plank system for movement of micro-organism population in confined environment
}

\author{
Jingyi Fu* $\quad$ Benoit Perthame ${ }^{\dagger} \quad$ Min Tang ${ }^{\ddagger}$
}

July 6,2021

\begin{abstract}
We consider self-propelled particles confined between two parallel plates, moving with a constant velocity while their moving direction changes by rotational diffusion. The probability distribution of such micro-organisms in confined environment is singular because particles accumulate at the boundaries. This leads us to distinguish between the probability distribution densities in the bulk and in the boundaries. They satisfy a degenerate Fokker-Planck system and we propose boundary conditions that take into account the switching between free-moving and boundary-contacting particles. Relative entropy property, a priori estimates and the convergence to an unique steady state are established. The steady states of both the PDE and individual based stochastic models are compared numerically.
\end{abstract}

2010 Mathematics Subject Classification. 35Q84; 35Q92; 35K65; 65C05; 65M06; 92C17

Keywords and phrases. Piecewise deterministic Markov process; Fokker-Planck equation; Relative entropy; Monte-Carlo simulations; Semi-discrete numerical schemes;

\section{Introduction}

Self-propelled micro-organisms, such as C. Crescentus [19, 20] and different types of Escherichia coli [1, 4, 22] accumulate near boundaries. Micro-swimmers bump into various obstacles and boundaries, alter the motility and are trapped in the near surface region. This phenomena strongly relates to the first stages of biofilm formation and is important when micro-organisms are moving inside micro-fluidic devices [7, 13, 15] or micro-structures [6, 23]. Two possible mechanisms are attributed to the near surface behavior, one is long range hydrodynamic interactions between swimming cell and the surface [1, 24], the other is steric interactions [19, 20, 12]. Models based on either of them can lead to accumulation at the boundaries.

Cell-wall interactions have attracted a lot of interest from micro-biologists $[1,4,9,14,19,20,22]$. In the experiment in [1], the authors measured the distribution of non-tumbling E. coli cells swimming between two parallel plates. They

*School of Mathematics, Institute of Natural Sciences and MOE-LSC, Shanghai Jiao Tong University, 200240, Shanghai. Email : nbfufu@sjtu.edu.cn

†Sorbonne Université, CNRS, Université de Paris, Inria, Laboratoire Jacques-Louis Lions UMR7598, F-75005 Paris. Email : Benoit.Perthame@sorbonne-universite.fr. B.P. has received funding from the European Research Council (ERC) under the European Union's Horizon 2020 research and innovation programme (grant agreement No 740623).

¥School of Mathematics, Institute of Natural Sciences and MOE-LSC, Shanghai Jiao Tong University, 200240, Shanghai. Email : tangmin@sjtu.edu.cn. M. Tang is supported by NSFC 11871340 and Young Changjiang Scholar of Ministry of Education in China. 
measured the cell density as a function of distance away from the plate. However, which of the two mechanisms, hydrodynamic or steric interactions, dominates the reorientation is not clear.

Motivated by the experiment in [1] and the theoretical models in [20], we consider here self-propelled micro-organisms confined between two parallel plates. We assume they move with a constant velocity $V$, while their moving direction changes by rotational diffusion and we ignore their interaction with the external fluid. More precisely, we consider a 2-dimensional stochastic model for particles confined between two horizontal boundaries $\{y= \pm L\}$. When the microorganism is away from boundaries, it swims at a constant speed $V$ and changes its orientation with white noise; and when it touches boundary, it keeps changing orientation with the same rotational Brownian motion but stays at the boundary until it is oriented away from the boundary. The evolution of its vertical position $Y_{t}$ and the angle between its orientation and horizontal plane $\Theta_{t}$, is described by a stochastic differential equation (SDE) entering the class of Piecewise-Deterministic Markov Processes [5],

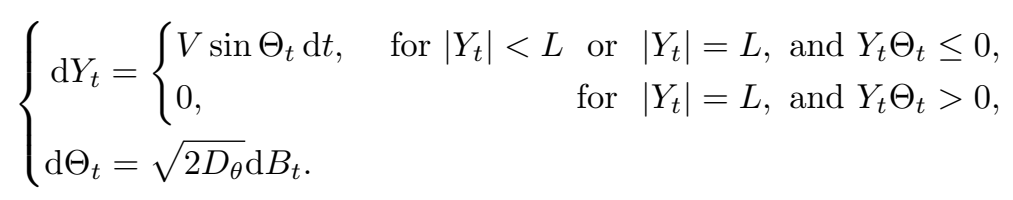

Here $B_{t}$ is a Brownian motion, and $D_{\theta}$ is the rotational diffusion coefficient. The process $\left(Y_{t}, \Theta_{t}\right)$ stays in the set $\Omega \cup \Omega_{+} \cup \Omega_{-}$. Here $\Omega=\{(y, \theta) \mid-L<y<L,-\pi<\theta<\pi\}$ in which cells can move freely with constant velocity $V$, i.e. cells are at free-swimming phase (FSP). When cells move towards the boundary and touch it, they keep propelling themselves but are not able to move forward due to the steric forces of the boundary. This state is called boundary contacting phase $(\mathrm{BCP})$ for which

$$
\left(Y_{t}, \Theta_{t}\right) \in\{(y, \theta) \mid y= \pm L, 0< \pm \theta<\pi\}=: \Omega_{ \pm}
$$

Then, cells return to FSP when their orientations are parallel to the boundary due to rotational diffusion. The mechanism of how cells change their movement directions near the boundary is more complex than rotational diffusion, for example the flow field close to the boundary plays an important role and some bacteria may tumble $[4,9,11,19]$. No-flux boundary condition for Fokker-Planck model is proposed in [12], and independently derived in [2] as the vanishing inertia limit of a elastic collision boundary condition. However, the no-flux condition represent the balance of translational diffusion and self-propulsion in the wall-normal direction. While in our model, only rotational diffusion is considered for simplicity, and the collision is assumed to be inelastic.

The SDE model (0.1) exhibits accumulations at the boundaries due to the direction persistence. The probability density function of the particles can be described by a Fokker-Plank equation inside $\left|Y_{t}\right|<L$, but particles behave differently at the boundaries and stop moving until the Brownian motion generates an angle forcing cells to re-enter the domain. The purpose of this work is to propose appropriate boundary conditions for the Fokker-Plank equation in confined environment, so that it can describe the correct probability density distribution of the SDE model (0.1). The boundary conditions are deduced from the switching between FSP and BCP which gives a coupled system of three equations, one for free swimming cells, the other two for boundary-contacting cells at $Y_{t}=L$ and $Y_{t}=-L$. Similar models have been proposed and studied before in [18, 26], in which semi-analytical solutions are derived for the steady state distribution. Here in this paper, we study the time evolutionary system and establish the relative entropy estimate and give its long term convergence to steady state solution.

The rest of this paper is organized as follows. In section 1, we introduce the Fokker-Plank model with appropriate boundary conditions when the micro-organisms are confined in two parallel plates. We give the relative entropy inequality and a priori estimate in section 2 and the weak convergence of the model system to steady state under certain assumptions in section 3. Finally, in section 4, we employ Von Mises iterations based on an entropy decreasing semi-discrete scheme to get the numerical solution of the steady state problem. We compare the numerical results with Monte-Carlo simulations. 


\section{The Fokker-Planck system}

We consider the following degenerate Fokker-Planck system:

$$
\left\{\begin{array}{lr}
\frac{\partial p}{\partial t}+V \sin \theta \frac{\partial p}{\partial y}-D_{\theta} \frac{\partial^{2} p}{\partial \theta^{2}}=0, & (y, \theta) \in \Omega \\
\frac{\partial p_{+}}{\partial t}-D_{\theta} \frac{\partial^{2} p_{+}}{\partial \theta^{2}}=V \sin \theta p(t, L, \theta), & (L, \theta) \in \Omega_{+} \\
\frac{\partial p_{-}}{\partial t}-D_{\theta} \frac{\partial^{2} p_{-}}{\partial \theta^{2}}=-V \sin \theta p(t,-L, \theta), & (-L, \theta) \in \Omega_{-}
\end{array}\right.
$$

Here $p(t, y, \theta)$ represents the probability distribution of free-swimming cells at time $t$, position $y$ and with moving direction $\theta ; p_{ \pm}(t, \theta)$ are the probability density distributions of boundary-contacting cells at time $t$, position $\pm L$ and with moving direction $\pm \theta \in(0, \pi)$. The diffusion in $\theta$ terms in (1.1a)-(1.1c) describe the rotational diffusion of cells while the $y$-derivative term models the free transport with velocity $V \sin \theta$ in $y$ direction when cells are at FSP. Notice the degeneracy of the system (the missing diffusion in $y$ ) which is standard in kinetic theory [25, 8, 3, 16]. The terms on the right hand side of (1.1b)-(1.1c) describe the transitions from FSP to BCP. Since when cells at FSP reach and move towards the boundary, they will switch to BCP, the changes $p_{ \pm}(t+\mathrm{d} t, \theta) \mathrm{d} \theta-p_{ \pm}(t, \theta) \mathrm{d} \theta$, with $\mathrm{d} t$ being a small time interval, are composed by two parts, one is due to the rotational diffusion and the other is from cells at FSP near $\pm L$. More precisely, let $L_{0}=L-V \sin \theta \mathrm{d} t$, the number of cells locating in the interval $\left[L_{0}, L\right.$ ) (or in $\left(-L,-L_{0}\right]$ for

$\left.p_{-}\right)$and moving forward in direction $\theta$ with velocity $V$ is $\int_{L_{0}}^{L} p(t, s, \theta) \mathrm{d} s$. When $\mathrm{d} t \rightarrow 0$, we find that the source terms for $p_{ \pm}$are as in (1.1b) and (1.1c). On the other hand, cells at BCP can switch to FSP when they are attached to the boundary at $y=L(y=-L)$ and change their swimming direction from $\theta \in(0, \pi)(\theta \in(-\pi, 0))$ to 0 or $\pi$ by rotational diffusion. Therefore the source term for $p$ inside $\Omega$ is 0 but there is flux coming from the boundary point $y= \pm L, \theta=0$ and $y=L, \theta=\pi, y=-L, \theta=-\pi$.

Boundary conditions. The Fokker-Planck system (1.1) comes with boundary conditions which we describe now. We begin with the equation for $p(t, y, \theta)$. On the one hand for $\theta$, cells at FSP moving with direction $\theta=\pi$ and $\theta=-\pi$ are the same and thus periodic boundary conditions are used

$$
p(t, y,-\pi)=p(t, y, \pi), \quad \frac{\partial p}{\partial \theta}(t, y,-\pi)=\frac{\partial p}{\partial \theta}(t, y, \pi), \quad-L<y<L .
$$

On the other hand for $y$ we have a transport term and we need boundary conditions only when cells enter the domain $(-L, L)$, and since there is no $\mathrm{BCP}$ cell in the entering boundary we impose Dirichlet condition

$$
p(t, L, \theta)=0, \quad \theta \in(-\pi, 0), \quad p(t,-L, \theta)=0, \quad \theta \in(0, \pi),
$$

Next, we turn to $p_{ \pm}$and their boundary condition related to diffusion. In order to express that BCP cells $p_{ \pm}$can only leave the boundary at $\theta=0$ or $\pm \pi$, we specify Dirichlet boundary conditions

$$
p_{+}(t, 0)=p_{+}(t, \pi)=0, \quad p_{-}(t, 0)=p_{-}(t,-\pi)=0 .
$$

It remains a difficulty related to the degeneracy of the Fokker-Planck equation. We need to express that BCP cells turning to the angles 0 or $\pm \pi$ will re-enter the domain, i.e., will change their status to FSP. 
To determine $p$ at $\pm L$, we need four conditions for $p(t, y, \theta)$ at the points $( \pm L, 0),( \pm L, \pm \pi)$. Since cells switch from BCP to FSP immediately when $\theta$ changes from positive to negative at $y=L$, from (1.1b), (1.1a), we have that, at $\theta=0$,

$$
\frac{\partial}{\partial t} \int_{0_{-}}^{0_{+}} p(t, L, \theta) \mathrm{d} \theta-\left.D_{\theta} \frac{\partial p}{\partial \theta}\right|_{\theta=0_{-}} ^{\theta=0_{+}}=-\left(\frac{\partial}{\partial t} \int_{0_{-}}^{0_{+}} p_{+}(t, \theta) \mathrm{d} \theta-\left.D_{\theta} \frac{\partial p_{+}}{\partial \theta}\right|_{\theta=0_{-}} ^{\theta=0_{+}}\right) \delta_{y=L}
$$

$p_{+}(t, \theta)$ is defined for $\theta \in(0, \pi)$, but it can be extended to $(-\pi, \pi)$ with $p_{+}(t, \theta)=0$ for $\theta \in(-\pi, 0)$, which indicates that $\frac{\partial p_{+}}{\partial \theta}\left(t, 0_{-}\right)=0$. Noting (1.4), we find

$$
D_{\theta} \frac{\partial p_{+}}{\partial \theta}\left(t, 0_{+}\right)+\left.D_{\theta} \int_{L-}^{L} \frac{\partial p}{\partial \theta} \mathrm{d} y\right|_{\theta=0_{-}} ^{\theta=0_{+}}=0
$$

Thus $\frac{\partial p}{\partial \theta}$ jumps at the point $(y, \theta)=(L, 0)$ while it is continuous at $(y, 0)$ with $y \in(-L, L)$. Similar calculations give the jumps of $\frac{\partial p}{\partial \theta}$ at $(y, \theta)=(-L, 0)$. Thus, we find

$$
\left.\frac{\partial p}{\partial \theta}(t, y, \theta)\right|_{\theta=0_{-}} ^{\theta=0_{+}}=-\frac{\partial p_{+}}{\partial \theta}\left(t, 0_{+}\right) \delta_{y=L}+\frac{\partial p_{-}}{\partial \theta}\left(t, 0_{-}\right) \delta_{y=-L}
$$

On the other hand, due to the periodic boundary conditions for $\theta$ in (1.2), by letting $p(t, y, \theta+2 \pi)=p(t, y, \theta)$ for $\theta \in[-\pi, 0)$, we can extend $p(t, y, \theta)$ from $\theta \in(-\pi, \pi)$ to $\theta \in(-\pi, 2 \pi)$. Then $\frac{\partial p}{\partial \theta}(t, y, \theta)$ is continuous at $\theta=\pi$ and $y \in(-L, L)$. Similar calculations as for $\theta=0$ yields

$$
\left.\frac{\partial p}{\partial \theta}(t, y, \theta)\right|_{\theta=\pi_{-}} ^{\theta=\pi_{+}}=\frac{\partial p_{+}}{\partial \theta}\left(t, \pi_{-}\right) \delta_{y=L}-\frac{\partial p_{-}}{\partial \theta}\left(t,-\pi_{+}\right) \delta_{y=-L}
$$

Remark 1.1. Another way of taking into account the degeneracy of the Fokker-Plank equation is to add a source term on the right hand side of the density equation for cells at FSP such that

$$
\frac{\partial p}{\partial t}+V \sin \theta \frac{\partial p}{\partial y}-D_{\theta} \frac{\partial^{2} p}{\partial \theta^{2}}=D_{\theta} S_{p}
$$

with the source term $S_{p}$ being given by

$$
S_{p}(t, y, \theta)=\delta_{y=L}\left[\delta_{\theta=0} \frac{\partial p_{+}}{\partial \theta}(t, 0)-\delta_{\theta=\pi} \frac{\partial p_{+}}{\partial \theta}(t, \pi)\right]+\delta_{y=-L}\left[-\delta_{\theta=0} \frac{\partial p_{-}}{\partial \theta}(t, 0)+\delta_{\theta=-\pi} \frac{\partial p_{-}}{\partial \theta}(t,-\pi)\right] .
$$

The source term can be understood as follows. Cells at BCP can switch to FSP when they are attached to the boundary at $y=L(y=-L)$ and change their swimming direction from $\theta \in(0, \pi)(\theta \in(-\pi, 0))$ to 0 or $\pi$ by rotational diffusion. The flux of a diffusion process is driven by the negative gradient. Therefore, $\frac{\partial p_{+}}{\partial \theta}(t, 0)$ is the number of cells that go outside of the interval $(0, \theta)$ by rotational diffusion at $y=L, \theta=0$. Cells switch from BCP to FSP immediately when $\theta$ becomes negative at $y=L$, which yields the source term $\delta_{y=L} \delta_{\theta=0} \frac{\partial p_{+}}{\partial \theta}(t, 0)$ in (1.8). Other terms in (1.8) can be understood similarly.

Mass conservation, weak form. Finally, we equip this system (1.1) with the following nonnegative bounded initial data

$$
p(0, y, \theta)=p_{0}(y, \theta), \quad(y, \theta) \in \Omega ; \quad p_{ \pm}(0, \theta)=p_{0, \pm}(\theta), \quad( \pm L, \theta) \in \Omega_{ \pm},
$$


The transfer terms and the boundary conditions are compatible with mass conservation, that means

$$
M(t)=\int_{-L}^{L} \int_{-\pi}^{\pi} p \mathrm{~d} \theta \mathrm{d} y+\int_{0}^{\pi} p_{+} \mathrm{d} \theta+\int_{-\pi}^{0} p_{-} \mathrm{d} \theta:=M(0) .
$$

We impose that the initial data satisfy $M(0)=1$.

More generally, mass conservation is a special case of the weak form of the equation. We define admissible test functions as smooth functions $\varphi(t, y, \theta)$ defined in $[0, T) \times \bar{\Omega}$ which satisfy the periodicity condition $\varphi(t, y,-\pi)=\varphi(t, y, \pi)$, $\frac{\partial \varphi}{\partial \theta}(t, y,-\pi)=\frac{\partial \varphi}{\partial \theta}(t, y, \pi), \varphi_{+}(t, \theta)$ defined on $[0, T) \times[0, \pi]$ and $\varphi_{-}(t, \theta)$ defined on $[0, T) \times[-\pi, 0]$ which satisfy $\varphi(t, L, \theta)=$ $\varphi_{+}(t, \theta)$, for $\theta \in[0, \pi]$ and $\varphi(t,-L, \theta)=\varphi_{-}(t, \theta)$ for $\theta \in[-\pi, 0]$. Then, the weak form is written, for all admissible test functions, as

$$
\begin{aligned}
\int_{0}^{T} \int_{\bar{\Omega}} & p\left[-\frac{\partial \varphi}{\partial t}-V \sin \theta \frac{\partial \varphi}{\partial y}-D_{\theta} \frac{\partial^{2} \varphi}{\partial \theta^{2}}\right]+\int_{0}^{T} \int_{-\pi}^{\pi} p_{+}\left[-\frac{\partial \varphi_{+}}{\partial t}-D_{\theta} \frac{\partial^{2} \varphi_{+}}{\partial \theta^{2}}\right]+\int_{0}^{T} \int_{\bar{\pi} \pi}^{\pi} p_{-}\left[-\frac{\partial \varphi_{-}}{\partial t}-D_{\theta} \frac{\partial^{2} \varphi_{-}}{\partial \theta^{2}}\right]= \\
& =\int_{\bar{\Omega}} p_{0} \varphi(t=0)+\int_{-\pi}^{\pi}\left[p_{0,+} \varphi_{+}(t=0)+p_{0,-} \varphi_{-}(t=0)\right]-\int_{\bar{\Omega}} p(T) \varphi(T)+\int_{-\pi}^{\pi}\left[p_{+}(T) \varphi_{+}(T)+p_{-}(T) \varphi_{-}(T)\right]
\end{aligned}
$$

We notice that the formal integration by parts uses all the boundary conditions.

This formulation and the a priori bounds in next section, also tells us that the probability distribution of the process (0.1) is composed of $p(t, y, \theta)$ which is absolutely continuous with respect to Lebesgue measure $\mathrm{d} y \mathrm{~d} \theta$, and singular parts, concentrated on the boundaries, $p_{ \pm}$which are absolutely continuous with respect to $\mathrm{d} \theta$.

\section{Relative entropy and a priori estimates}

Since the model in (1.1) is linear, we take for granted existence and non-negativity of weak solutions even if its degeneracy and boundary conditions yield interesting mathematical questions. We derive here formally the relative entropy estimate and we deduce some a priori bounds (see [21] for the general form of entropy structure).

The relative entropy uses the stationary state distributions $q(y, \theta)$ and $q_{ \pm}(\theta)$ which satisfy:

$$
\left\{\begin{array}{lr}
-D_{\theta} \frac{\partial^{2} q}{\partial \theta^{2}}+V \sin \theta \frac{\partial q}{\partial y}=0, & (y, \theta) \in \Omega \\
-D_{\theta} \frac{\mathrm{d}^{2} q_{+}}{\mathrm{d} \theta^{2}}=V \sin \theta q(L, \theta), & (L, \theta) \in \Omega_{+} \\
-D_{\theta} \frac{\mathrm{d}^{2} q_{-}}{\mathrm{d} \theta^{2}}=-V \sin \theta q(-L, \theta), & (-L, \theta) \in \Omega_{-}
\end{array}\right.
$$

with the boundary conditions

$$
\left\{\begin{array}{l}
q(y, \theta)=q(y, \theta+2 \pi), \quad \frac{\partial q}{\partial \theta}(y, \theta)=\frac{\partial q}{\partial \theta}(y, \theta+2 \pi), \quad(y, \theta) \in \Omega \\
q(L, \theta)=0, \quad \theta \in(-\pi, 0), \quad q(-L, \theta)=0, \quad \theta \in(0, \pi) \\
\left.\frac{\partial q}{\partial \theta}(y, \theta)\right|_{\theta=0_{-}} ^{\theta=0_{+}}=-\frac{\partial q_{+}}{\partial \theta}\left(0_{+}\right) \delta_{y=L}+\frac{\partial q_{-}}{\partial \theta}\left(0_{-}\right) \delta_{y=-L} \\
\left.\frac{\partial q}{\partial \theta}(y, \theta)\right|_{\theta=\pi_{+}} ^{\theta=\pi_{+}}=\frac{\partial q_{+}}{\partial \theta}\left(\pi_{-}\right) \delta_{y=L}-\frac{\partial q_{-}}{\partial \theta}\left(-\pi_{+}\right) \delta_{y=-L} \\
q_{+}(0)=q_{+}(\pi)=0, \quad q_{-}(0)=q_{-}(-\pi)=0
\end{array}\right.
$$


Since the model is linear, we can normalize $\left(q, q_{ \pm}\right)$and let it satisfy

$$
\int_{-L}^{L} \int_{-\pi}^{\pi} q \mathrm{~d} \theta \mathrm{d} y+\int_{0}^{\pi} q_{+} \mathrm{d} \theta+\int_{-\pi}^{0} q_{-} \mathrm{d} \theta=1
$$

Thanks to the linearity of the model, and despite its degeneracy, we admit well-posedness and non-negativity of the solution to (2.1)-(2.3). Moreover, we also admit the strong maximum principle and get that $q$ is strictly positive in the domain $(-L, L) \times(-\pi, \pi)$. Therefore, $\Omega_{0}=\{(y, \theta) \in \Omega \mid q(y, \theta)=0\}$ is restricted to the incoming part of the boundary. Moreover, concavity of $q_{ \pm}$guarantees their strictly positivity in $\Omega_{ \pm}$.

Before establishing the relative entropy estimate, we define formally the relative gap $\omega$ and $\omega_{ \pm}$as

$$
\begin{cases}\omega(t, y, \theta)=\frac{p(t, y, \theta)}{q(y, \theta)}-1, & (y, \theta) \in \Omega \backslash \Omega_{0}, \\ \omega_{+}(t, \theta)=\frac{p_{+}(t, \theta)}{q_{+}(\theta)}-1, & (L, \theta) \in \Omega_{+}, \\ \omega_{-}(t, \theta)=\frac{p_{-}(t, \theta)}{q_{-}(\theta)}-1, & (-L, \theta) \in \Omega_{-} .\end{cases}
$$

For the sake of simplicity, hereafter we denote $\omega(t, \pm L, \theta)$ by $\omega_{ \pm L}(t, \theta), p(t, \pm L, \theta)$ by $p_{ \pm L}(t, \theta)$, and $q(t, \pm L, \theta)$ by $q_{ \pm L}(\theta)$. We have the following relative entropy inequality:

Theorem 2.1. Consider a solution $p$ to equation (1.1) with the boundary conditions in (1.2) and $q$ is a solution to (2.1) with the boundary conditions in (2.2). For any convex function $H \in \mathcal{C}^{2}(\mathbb{R})$, the relative gaps $\omega$ and $\omega_{ \pm}$as in (2.4) satisfy

$$
\frac{\mathrm{d}}{\mathrm{d} t}\left[\int_{-L}^{L} \int_{-\pi}^{\pi} q H(\omega) \mathrm{d} \theta \mathrm{d} y+\int_{0}^{\pi} q_{+} H\left(\omega_{+}\right) \mathrm{d} \theta+\int_{-\pi}^{0} q_{-} H\left(\omega_{-}\right) \mathrm{d} \theta\right]=-D_{H}^{(1)}(t)-D_{H}^{(2)}(t)-D_{H}^{(3)}(t) \leq 0
$$

where the dissipation from diffusion is,

$$
D_{H}^{(1)}(t)=D_{\theta} \int_{0}^{\pi} q_{+} H^{\prime \prime}\left(\omega_{+}\right)\left(\frac{\partial \omega_{+}}{\partial \theta}\right)^{2} \mathrm{~d} \theta+D_{\theta} \int_{-\pi}^{0} q_{-} H^{\prime \prime}\left(\omega_{-}\right)\left(\frac{\partial \omega_{-}}{\partial \theta}\right)^{2} \mathrm{~d} \theta+D_{\theta} q \int_{-L}^{L} \int_{-\pi}^{\pi}\left[q H^{\prime \prime}(\omega)\left(\frac{\partial \omega}{\partial \theta}\right)^{2}\right] \mathrm{d} \theta \mathrm{d} y
$$

the dissipation of switching from FSP to $B C P$ is

$$
\begin{aligned}
D_{H}^{(2)}(t)= & \int_{0}^{\pi} V \sin \theta q_{+L}\left[H\left(\omega_{+L}\right)-H\left(\omega_{+}\right)-H^{\prime}\left(\omega_{+}\right)\left(\omega_{+L}-\omega_{+}\right)\right] \mathrm{d} \theta \\
& +\int_{-\pi}^{0}-V \sin \theta q_{-L}\left[H\left(\omega_{-L}\right)-H\left(\omega_{-}\right)-H^{\prime}\left(\omega_{-}\right)\left(\omega_{-L}-\omega_{-}\right)\right] \mathrm{d} \theta
\end{aligned}
$$

and the dissipation of switching from BCP to FSP is

$$
\begin{aligned}
D_{H}^{(3)}(t)= & -\left.D_{\theta}\left\{\left[H\left(\omega_{+}\right)-H\left(\omega_{+L}\right)-H^{\prime}\left(\omega_{+L}\right)\left(\omega_{+}-\omega_{+L}\right)\right] \frac{\partial q_{+}}{\partial \theta}\right\}\right|_{0} ^{\pi} \\
& -\left.D_{\theta}\left\{\left[H\left(\omega_{-}\right)-H\left(\omega_{-L}\right)-H^{\prime}\left(\omega_{-L}\right)\left(\omega_{-}-\omega_{-L}\right)\right] \frac{\partial q_{-}}{\partial \theta}\right\}\right|_{-\pi} ^{0} .
\end{aligned}
$$


Proof. Substituting $p_{+}=q_{+} \omega_{+}+q_{+}$into (1.1b) and using (2.1b), we get

$$
\begin{aligned}
q_{+} \frac{\partial \omega_{+}}{\partial t} & =D_{\theta}\left(1+\omega_{+}\right) \frac{\partial^{2} q_{+}}{\partial \theta^{2}}+2 D_{\theta} \frac{\partial q_{+}}{\partial \theta} \frac{\partial \omega_{+}}{\partial \theta}+D_{\theta} q_{+} \frac{\partial^{2} \omega_{+}}{\partial \theta^{2}}+V \sin \theta q_{+L}\left(1+\omega_{+L}\right) \\
& =D_{\theta}\left[\left(\omega_{+}-\omega_{+L}\right) \frac{\partial^{2} q_{+}}{\partial \theta^{2}}+2 \frac{\partial q_{+}}{\partial \theta} \frac{\partial \omega_{+}}{\partial \theta}+q_{+} \frac{\partial^{2} \omega_{+}}{\partial \theta^{2}}\right] .
\end{aligned}
$$

Multiplying both sides of (2.9) by $H^{\prime}\left(\omega_{+}\right)$yields

$$
\begin{aligned}
\frac{q_{+}}{D_{\theta}} \frac{\partial H\left(\omega_{+}\right)}{\partial t} & =H^{\prime}\left(\omega_{+}\right)\left(\omega_{+}-\omega_{+L}\right) \frac{\partial^{2} q_{+}}{\partial \theta^{2}}+2 \frac{\partial q_{+}}{\partial \theta} \frac{\partial H\left(\omega_{+}\right)}{\partial \theta}+q_{+} H^{\prime}\left(\omega_{+}\right) \frac{\partial^{2} \omega_{+}}{\partial \theta^{2}} \\
& =H^{\prime}\left(\omega_{+}\right)\left(\omega_{+}-\omega_{+L}\right) \frac{\partial^{2} q_{+}}{\partial \theta^{2}}+2 \frac{\partial q_{+}}{\partial \theta} \frac{\partial H\left(\omega_{+}\right)}{\partial \theta}+q_{+}\left[\frac{\partial^{2} H\left(\omega_{+}\right)}{\partial \theta^{2}}-H^{\prime \prime}\left(\omega_{+}\right)\left(\frac{\partial \omega_{+}}{\partial \theta}\right)^{2}\right] .
\end{aligned}
$$

Combining the above equation with

$$
\frac{\partial^{2}\left(q_{+} H\left(\omega_{+}\right)\right)}{\partial \theta^{2}}=H\left(\omega_{+}\right) \frac{\partial^{2} q_{+}}{\partial \theta^{2}}+2 \frac{\partial q_{+}}{\partial \theta} \frac{\partial H\left(\omega_{+}\right)}{\partial \theta}+q_{+} \frac{\partial^{2} H\left(\omega_{+}\right)}{\partial \theta^{2}},
$$

we find

$$
\begin{aligned}
\left(\frac{\partial}{\partial t}-D_{\theta} \frac{\partial^{2}}{\partial \theta^{2}}\right)\left[q_{+} H\left(\omega_{+}\right)\right]= & D_{\theta}\left[H^{\prime}\left(\omega_{+}\right)\left(\omega_{+}-\omega_{+L}\right)-H\left(\omega_{+}\right)\right] \frac{\partial^{2} q_{+}}{\partial \theta^{2}}-D_{\theta} q_{+} H^{\prime \prime}\left(\omega_{+}\right)\left(\frac{\partial \omega_{+}}{\partial \theta}\right)^{2} \\
= & V \sin \theta q_{+L} H\left(\omega_{+L}\right)-V \sin \theta q_{+L}\left[H\left(\omega_{+L}\right)-H\left(\omega_{+}\right)-H^{\prime}\left(\omega_{+}\right)\left(\omega_{+L}-\omega_{+}\right)\right] \\
& -D_{\theta} q_{+} H^{\prime \prime}\left(\omega_{+}\right)\left(\frac{\partial \omega_{+}}{\partial \theta}\right)^{2} .
\end{aligned}
$$

Similarly, the equation for $q_{-} H\left(\omega_{-}\right)$is

$$
\begin{aligned}
\left(\frac{\partial}{\partial t}-D_{\theta} \frac{\partial^{2}}{\partial \theta^{2}}\right)\left[q_{-} H\left(\omega_{-}\right)\right]= & -V \sin \theta q_{-L} H\left(\omega_{-L}\right)+V \sin \theta q_{-L}\left[H\left(\omega_{-L}\right)-H\left(\omega_{-}\right)-H^{\prime}\left(\omega_{-}\right)\left(\omega_{-L}-\omega_{-}\right)\right] \\
& -D_{\theta} q_{-} H^{\prime \prime}\left(\omega_{-}\right)\left(\frac{\partial \omega_{-}}{\partial \theta}\right)^{2} .
\end{aligned}
$$

Substituting $p=q \omega+q$ into (1.1a) and using (2.1a), we have

$$
\begin{aligned}
q \frac{\partial \omega}{\partial t} & =(1+\omega)\left(D_{\theta} \frac{\partial^{2} q}{\partial \theta^{2}}-V \sin \theta \frac{\partial q}{\partial y}\right)+2 D_{\theta} \frac{\partial q}{\partial \theta} \frac{\partial \omega}{\partial \theta}+D_{\theta} q \frac{\partial^{2} \omega}{\partial \theta^{2}}-V \sin \theta q \frac{\partial \omega}{\partial y} \\
& =2 D_{\theta} \frac{\partial q}{\partial \theta} \frac{\partial \omega}{\partial \theta}+D_{\theta} q \frac{\partial^{2} \omega}{\partial \theta^{2}}-V \sin \theta q \frac{\partial \omega}{\partial y} .
\end{aligned}
$$

Multiplying both sides of the above equation by $H^{\prime}(\omega)$ gives

$$
\begin{aligned}
\frac{\partial(q H(\omega))}{\partial t} & =2 D_{\theta} \frac{\partial q}{\partial \theta} \frac{\partial H(\omega)}{\partial \theta}+D_{\theta} q H^{\prime}(\omega) \frac{\partial^{2} \omega}{\partial \theta^{2}}-V \sin \theta q \frac{\partial H(\omega)}{\partial y} \\
& =2 D_{\theta} \frac{\partial q}{\partial \theta} \frac{\partial H(\omega)}{\partial \theta}+D_{\theta} q\left[\frac{\partial^{2} H(\omega)}{\partial \theta^{2}}-H^{\prime \prime}(\omega)\left(\frac{\partial \omega}{\partial \theta}\right)^{2}\right]-V \sin \theta q \frac{\partial H(\omega)}{\partial y} \\
& =D_{\theta} \frac{\partial^{2}(q H(\omega))}{\partial \theta^{2}}-D_{\theta} H(\omega) \frac{\partial^{2} q}{\partial \theta^{2}}-D_{\theta} q H^{\prime \prime}(\omega)\left(\frac{\partial \omega}{\partial \theta}\right)^{2}-V \sin \theta\left[\frac{\partial(q H(\omega))}{\partial y}-H(\omega) \frac{\partial q}{\partial y}\right] \\
& =D_{\theta} \frac{\partial^{2}(q H(\omega))}{\partial \theta^{2}}-V \sin \theta \frac{\partial(q H(\omega))}{\partial y}-D_{\theta} q H^{\prime \prime}(\omega)\left(\frac{\partial \omega}{\partial \theta}\right)^{2} .
\end{aligned}
$$


The equality in (2.5) can be obtained by rearranging $\int_{0_{+}}^{\pi_{-}}(2.11) \mathrm{d} \theta+\int_{-\pi_{+}}^{0_{-}}(2.12) \mathrm{d} \theta+\int_{-L}^{L} \int_{-\pi_{+}}^{0_{-}}(2.14) \mathrm{d} \theta \mathrm{d} y+\int_{-L}^{L} \int_{0_{+}}^{\pi_{-}}$ (2.14) $\mathrm{d} \theta \mathrm{d} y$. The two terms $D_{H}^{(1)}$ and $D_{H}^{(2)}$ are obvious and $D_{H}^{(3)}$ is from the diffusion terms. After integrating and using (1.3), the second order derivative in $\theta$ terms becomes

$$
D_{\theta}\left[\left.\frac{\partial\left(q_{+} H\left(\omega_{+}\right)\right)}{\partial \theta}\right|_{\theta=0_{+}} ^{\theta=\pi_{-}}+\left.\frac{\partial\left(q_{-} H\left(\omega_{-}\right)\right)}{\partial \theta}\right|_{\theta=-\pi_{+}} ^{\theta=0_{-}}+\left.\int_{-L}^{L} \frac{\partial(q H(\omega))}{\partial \theta}\right|_{\theta=0_{+}} ^{\theta=\pi_{-}} \mathrm{d} y+\left.\int_{-L}^{L} \frac{\partial(q H(\omega))}{\partial \theta}\right|_{\theta=-\pi_{+}} ^{\theta=0} \mathrm{~d} y\right]
$$

From the definitions of $\omega, \omega_{ \pm}$in $(2.4)$, we have

$$
p=\omega q+q, \quad p_{ \pm}=\omega_{ \pm} q_{ \pm}+q_{ \pm}
$$

Then using the continuity of $q$ and $\omega$ for $(y, \theta) \in(-L, L) \times\{0\},(1.3),(1.5)$ and second and third boundary conditions in $(2.2)$, we find

$$
\begin{aligned}
\left.\int_{-L}^{L} \frac{\partial(q H(\omega))}{\partial \theta}\right|_{\theta=0_{-}} ^{\theta=0_{+}} \mathrm{d} y= & \left.\int_{-L}^{L} H^{\prime}(\omega) \frac{\partial(\omega q)}{\partial \theta}\right|_{\theta=0_{-}} ^{\theta=0_{+}} \mathrm{d} y+\left.\int_{-L}^{L}\left(H(\omega)-\omega H^{\prime}(\omega)\right) \frac{\partial q}{\partial \theta}\right|_{\theta=0_{-}} ^{\theta=0_{+}} \mathrm{d} y \\
= & \left.\int_{-L}^{L} H^{\prime}(\omega) \frac{\partial p}{\partial \theta}\right|_{\theta=0_{-}} ^{\theta=0_{+}} \mathrm{d} y+\left.\int_{-L}^{L}\left(H(\omega)-(\omega+1) H^{\prime}(\omega)\right) \frac{\partial q}{\partial \theta}\right|_{\theta=0_{-}} ^{\theta=0_{+}} \mathrm{d} y \\
= & -\int_{-L}^{L} H^{\prime}(\omega(t, y, 0)) \frac{\partial p_{+}}{\partial \theta}\left(t, 0_{+}\right) \delta_{y=L} \mathrm{~d} y+\int_{-L}^{L} H^{\prime}(\omega(t, y, 0)) \frac{\partial p_{-}}{\partial \theta}\left(t, 0_{-}\right) \delta_{y=-L} \mathrm{~d} y \\
& -\int_{-L}^{L}\left[H(\omega(t, y, 0))-(\omega(t, y, 0)+1) H^{\prime}(\omega(t, y, 0))\right] \frac{\partial q_{+}}{\partial \theta}\left(0_{+}\right) \delta_{y=L} \mathrm{~d} y \\
& +\int_{-L}^{L}\left[H(\omega(t, y, 0))-(\omega(t, y, 0)+1) H^{\prime}(\omega(t, y, 0))\right] \frac{\partial q_{-}}{\partial \theta}\left(0_{-}\right) \delta_{y=-L} \mathrm{~d} y
\end{aligned}
$$

which we also write

$$
\begin{aligned}
= & -H^{\prime}\left(\omega_{+L}(t, 0)\right)\left[q_{+}\left(0_{+}\right) \frac{\partial \omega_{+}}{\partial \theta}\left(t, 0_{+}\right)+\left(\omega_{+}\left(t, 0_{+}\right)+1\right) \frac{\partial q_{+}}{\partial \theta}\left(0_{+}\right)\right] \\
& +H^{\prime}\left(\omega_{-L}(t, 0)\right)\left[q_{-}\left(0_{-}\right) \frac{\partial \omega_{-}}{\partial \theta}\left(t, 0_{-}\right)+\left(\omega_{-}\left(t, 0_{-}\right)+1\right) \frac{\partial q_{-}}{\partial \theta}\left(0_{-}\right)\right] \\
& -\left[H\left(\omega_{+L}(t, 0)\right)-\left(\omega_{+L}(t, 0)+1\right) H^{\prime}\left(\omega_{+L}(t, 0)\right)\right] \frac{\partial q_{+}}{\partial \theta}\left(0_{+}\right) \\
& +\left[H\left(\omega_{-L}(t, 0)\right)-\left(\omega_{-L}(t, 0)+1\right) H^{\prime}\left(\omega_{-L}(t, 0)\right)\right] \frac{\partial q_{-}}{\partial \theta}\left(0_{-}\right) \\
= & -H^{\prime}\left(\omega_{+L}(t, 0)\right) q_{+}\left(0_{+}\right) \frac{\partial \omega_{+}}{\partial \theta}\left(t, 0_{+}\right)-\left(\omega_{+}\left(t, 0_{+}\right)-\omega_{+L}\left(t, 0_{+}\right)\right) H^{\prime}\left(\omega_{+L}(t, 0)\right) \frac{\partial q_{+}}{\partial \theta}\left(0_{+}\right)-H\left(\omega_{+L}(t, 0)\right) \frac{\partial q_{+}}{\partial \theta}\left(0_{+}\right) \\
& +H^{\prime}\left(\omega_{-L}(t, 0)\right) q_{-}\left(0_{-}\right) \frac{\partial \omega_{-}}{\partial \theta}\left(t, 0_{-}\right)+\left(\omega_{-}\left(t, 0_{-}\right)-\omega_{-L}\left(t, 0_{-}\right)\right) H^{\prime}\left(\omega_{-L}(t, 0)\right) \frac{\partial q_{-}}{\partial \theta}\left(0_{-}\right)+H\left(\omega_{-L}(t, 0)\right) \frac{\partial q_{-}}{\partial \theta}(0-) .
\end{aligned}
$$


Then using (1.4) and (2.2), we obtain

$$
\begin{aligned}
& -\frac{\partial\left(q_{+} H\left(\omega_{+}\right)\right)}{\partial \theta}\left(t, 0_{+}\right)+\frac{\partial\left(q_{-} H\left(\omega_{-}\right)\right)}{\partial \theta}\left(t, 0_{-}\right)-\left.\int_{-L}^{L} \frac{\partial(q H(\omega))}{\partial \theta}\right|_{\theta=0_{-}} ^{\theta=0_{+}} \mathrm{d} y \\
= & {\left[H\left(\omega_{+L}\left(t, 0_{+}\right)\right)-H\left(\omega_{+}\left(t, 0_{+}\right)\right)-H^{\prime}\left(\omega_{+L}\left(t, 0_{+}\right)\right)\left(\omega_{+L}\left(t, 0_{+}\right)-\omega_{+}\left(t, 0_{+}\right)\right)\right] \frac{\partial q_{+}}{\partial \theta}\left(0_{+}\right) } \\
& -\left[H\left(\omega_{-L}\left(t, 0_{-}\right)\right)-H\left(\omega_{-}\left(t, 0_{-}\right)\right)-H^{\prime}\left(\omega_{-L}\left(t, 0_{-}\right)\right)\left(\omega_{-L}\left(t, 0_{-}\right)-\omega_{-}\left(t, 0_{-}\right)\right)\right] \frac{\partial q_{-}}{\partial \theta}\left(0_{-}\right) .
\end{aligned}
$$

The other terms in (2.15) can be reformulated similarly and we obtain $D_{H}^{(3)}$. Due to the positivity of $q_{ \pm}$and the boundary conditions that $q_{ \pm}(0)=q_{ \pm}( \pm \pi)=0$, we have $\frac{\partial q_{+}}{\partial \theta}(0), \frac{\partial q_{-}}{\partial \theta}(-\pi)>0$ and $\frac{\partial q_{+}}{\partial \theta}(\pi), \frac{\partial q_{-}}{\partial \theta}(0)<0$. Then convexity of $H$ and the positivity $q_{ \pm}, q$ give the positivity of $D_{H}^{(1)}, D_{H}^{(2)}, D_{H}^{(3)}$. This concludes the derivation of the relative entropy inequality.

The immediate consequence of relative entropy inequality (2.5) is the following a priori estimate which indicates that if initially $p, p_{ \pm}$are bounded, they will not blow up.

Corollary 2.2. Assume there exists a constant $\Lambda>0$ such that

$$
\|\omega(0, \cdot, \cdot)\|_{L^{\infty}(\Omega)} \leq \Lambda, \quad\left\|\omega_{ \pm}(0, \cdot)\right\|_{L^{\infty}\left(\Omega_{ \pm}\right)} \leq \Lambda
$$

then for all $t \geq 0$,

$$
\|\omega(t, \cdot, \cdot)\|_{L^{\infty}(\Omega)} \leq \Lambda, \quad\left\|\omega_{ \pm}(t, \cdot)\right\|_{L^{\infty}\left(\Omega_{ \pm}\right)} \leq \Lambda .
$$

Proof. By choosing the convex function $H(x)=\left[(x-\Lambda)_{+}\right]^{4} \in \mathcal{C}^{2}(\mathbb{R})$ in $(2.5)$, we find

$$
\frac{\mathrm{d}}{\mathrm{d} t}\left[\int_{-L}^{L} \int_{-\pi}^{\pi} q(\omega-\Lambda)_{+}^{4} \mathrm{~d} \theta \mathrm{d} y+\int_{0}^{\pi} q_{+}\left(\omega_{+}-\Lambda\right)_{+}^{4} \mathrm{~d} \theta+\int_{-\pi}^{0} q_{-}\left(\omega_{-}-\Lambda\right)_{+}^{4} \mathrm{~d} \theta\right] \leq 0 .
$$

Then, we conclude that

$$
\begin{aligned}
& \int_{-L}^{L} \int_{-\pi}^{\pi} q(\omega(T, y, \theta)-\Lambda)_{+}^{4} \mathrm{~d} \theta \mathrm{d} y+\int_{0}^{\pi} q_{+}\left(\omega_{+}(T, \theta)-\Lambda\right)_{+}^{4} \mathrm{~d} \theta+\int_{-\pi}^{0} q_{-}\left(\omega_{-}(T, \theta)-\Lambda\right)_{+}^{4} \mathrm{~d} \theta \\
& \quad \leq \int_{-L}^{L} \int_{-\pi}^{\pi} q(\omega(0, y, \theta)-\Lambda)_{+}^{4} \mathrm{~d} \theta \mathrm{d} y+\int_{0}^{\pi} q_{+}\left(\omega_{+}(0, \theta)-\Lambda\right)_{+}^{4} \mathrm{~d} \theta+\int_{-\pi}^{0} q_{-}\left(\omega_{-}(0, \theta)-\Lambda\right)_{+}^{4} \mathrm{~d} \theta=0 .
\end{aligned}
$$

Since $q$ is strictly positive for all $(y, \theta)$ in $\Omega$ except a zero measure subset, and $q_{ \pm}$are strictly positive for $\theta \in \Omega_{ \pm}$, we conclude the proof.

\section{Long time convergence}

Another consequence of the relative entropy is the weak convergence to steady state for solutions of (1.1), under certain assumptions. The approach is as follows. For $k \in \mathbb{N}$, we define the time shifts

$$
\omega_{k}(t, y, \theta)=\omega(t+k, y, \theta), \quad(y, \theta) \in \Omega ; \quad \omega_{k, \pm}(t, \theta)=\omega_{ \pm}(t+k, \theta), \quad( \pm L, \theta) \in \Omega_{ \pm} .
$$


From Corollary 2.2, $\left\{\omega_{k}\right\},\left\{\omega_{k, \pm}\right\}$ are uniformly bounded in $L^{\infty}$. By weak compactness of $L^{\infty}$ space, there exists a subsequence $\left\{n_{k}\right\}_{k \in \mathbb{N}}$ and limit functions $\omega_{\infty} \in L^{\infty}(\mathbb{R} \times \Omega), \omega_{\infty, \pm} \in L^{\infty}\left(\mathbb{R} \times \Omega_{ \pm}\right)$, such that for all functions $\phi(t, y, \theta)$ in $L^{1}(\mathbb{R} \times \Omega)$ and $\phi_{ \pm}(t, \theta)$ in $L^{1}\left(\mathbb{R} \times \Omega_{ \pm}\right)$, we have for all $T \in \mathbb{R}$,

$$
\begin{aligned}
\lim _{n_{k} \rightarrow \infty} \int_{T}^{\infty} \int_{-L}^{L} \int_{-\pi}^{\pi} \phi \omega_{n_{k}} \mathrm{~d} \theta \mathrm{d} y \mathrm{~d} t & =\int_{T}^{\infty} \int_{-L}^{L} \int_{-\pi}^{\pi} \phi \omega_{\infty} \mathrm{d} \theta \mathrm{d} y \mathrm{~d} t \\
\lim _{n_{k} \rightarrow \infty} \int_{T}^{\infty} \int_{0}^{ \pm \pi} \phi_{ \pm} \omega_{n_{k}, \pm} \mathrm{d} \theta \mathrm{d} t & =\int_{T}^{\infty} \int_{0}^{ \pm \pi} \phi_{ \pm} \omega_{\infty, \pm} \mathrm{d} \theta \mathrm{d} t
\end{aligned}
$$

We prove in the subsequent part that $\omega_{\infty}, \omega_{\infty, \pm}$ are zero functions.

Theorem 3.1. Assume that the initial data $\omega(0, \cdot, \cdot)$ and $\omega_{ \pm}(0, \cdot)$ are uniformly bounded in $L^{\infty}$, that is (2.16) are satisfied. Then

$$
\omega_{\infty}=0, \quad \omega_{\infty, \pm}=0, \quad \text { a.e. }
$$

and thus for any test function $\phi(y, \theta) \in L^{1}(\Omega), \phi_{+}(\theta) \in L^{1}(0, \pi), \phi_{-}(\theta) \in L^{1}(-\pi, 0)$,

$$
\lim _{t \rightarrow+\infty} \int_{-\pi}^{\pi} \int_{-L}^{L} \omega(t, y, \theta) \phi(y, \theta) \mathrm{d} y \mathrm{~d} \theta=\lim _{t \rightarrow+\infty} \int_{-\pi}^{0} \omega_{-}(t, \theta) \phi_{-}(\theta) \mathrm{d} \theta=\lim _{t \rightarrow+\infty} \int_{0}^{\pi} \omega_{+}(t, \theta) \phi_{+}(\theta) \mathrm{d} \theta=0 .
$$

Proof. The proof is divided into five steps, we first show that $\omega_{\infty}, \omega_{\infty, \pm}$ are independent of $\theta$ by the relative entropy estimate, then prove their independence of time and $y$ by the equation and finally we use the conservation of mass to show that $\omega_{\infty}, \omega_{\infty, \pm}$ have to be zero almost everywhere.

First step: strong convergence from relative entropy inequality $(2.5)$. Using the relative entropy $H(x)=x^{2}$ in $(2.5)$, we obtain

$$
I(t)=\int_{-L}^{L} \int_{-\pi}^{\pi} q \omega^{2} \mathrm{~d} \theta \mathrm{d} y+\int_{0}^{\pi} q_{+} \omega_{+}^{2} \mathrm{~d} \theta+\int_{-\pi}^{0} q_{-} \omega_{-}^{2} \mathrm{~d} \theta \quad \text { and } \quad \frac{\mathrm{d} I(t)}{\mathrm{d} t} \leq 0 .
$$

Since the initial data are bounded as in (2.16), $I(0) \leq(4 \pi L+2 \pi) \Lambda^{2}<\infty$. Then, since $I(t)$ is nonnegative, it converges to a limit when $t \rightarrow \infty$. Therefore, $\lim _{\tau \rightarrow \infty} \int_{\tau}^{\infty} \frac{\mathrm{d} I(t)}{\mathrm{d} t} \mathrm{~d} t=0$ and we find

$$
\lim _{\tau \rightarrow \infty} \int_{\tau}^{\infty}\left(D_{H}^{(1)}+D_{H}^{(2)}+D_{H}^{(3)}\right)\left[\omega, \omega_{ \pm}\right] \mathrm{d} t=0
$$

For $\omega_{n_{k}}$ and $\omega_{n_{k}, \pm}$, this gives us

$$
\lim _{n_{k} \rightarrow \infty} \int_{T}^{\infty}\left(D_{H}^{(1)}+D_{H}^{(2)}+D_{H}^{(3)}\right)\left[\omega_{n_{k}}, \omega_{n_{k}, \pm}\right](t) \mathrm{d} t=\lim _{n_{k} \rightarrow \infty} \int_{T+n_{k}}^{\infty}\left(D_{H}^{(1)}+D_{H}^{(2)}+D_{H}^{(3)}\right)\left[\omega, \omega_{ \pm}\right](t) \mathrm{d} t=0 .
$$

Consequently, due to the non-negativity of each term in $D_{H}^{(1)}, D_{H}^{(2)}, D_{H}^{(3)}$ in $(2.6)-(2.8)$, we can extract a subsequence, still denoted by $n_{k}$ such that

$$
\begin{cases}\frac{\partial \omega_{\infty}}{\partial \theta}(t, y, \theta)=0, & \text { a.e. in }[T, \infty) \times \Omega, \\ \frac{\partial \omega_{\infty, \pm}}{\partial \theta}(t, \theta)=0, & \text { a.e. in }[T, \infty) \times \Omega_{ \pm}, \\ \lim _{n_{k} \rightarrow \infty} \omega_{n_{k}, \pm L}(t, \theta)-\omega_{n_{k}, \pm}(t, \theta)=0, & \text { a.e. in }[T, \infty) \times \Omega_{ \pm}, \\ \lim _{n_{k} \rightarrow \infty} \omega_{n_{k}, \pm L}(t, 0)-\omega_{n_{k}, \pm}(t, 0)=0, & \text { a.e. in }[T, \infty), \\ \lim _{n_{k} \rightarrow \infty} \omega_{n_{k}, \pm L}(t, \pm \pi)-\omega_{n_{k}, \pm}(t, \pm \pi)=0, & \text { a.e. in }[T, \infty) .\end{cases}
$$


Second step: the limits $\omega_{\infty, \pm}$ are independent of time. Since $q_{ \pm}$are independent of time, (2.9) indicates that, for all $k \in \mathbb{N}, \omega_{n_{k}}$ and $\omega_{n_{k},+}$ satisfy

$$
q_{+} \frac{\partial \omega_{n_{k},+}}{\partial t}=D_{\theta}\left(\left(\omega_{n_{k},+}-\omega_{n_{k},+L}\right) \frac{\partial^{2} q_{+}}{\partial \theta^{2}}+2 \frac{\partial q_{+}}{\partial \theta} \frac{\partial \omega_{n_{k},+}}{\partial \theta}+q_{+} \frac{\partial^{2} \omega_{n_{k},+}}{\partial \theta^{2}}\right), \quad \theta \in(0, \pi) .
$$

We choose compactly supported test function $\phi_{+}(t, \theta) \in L^{1}\left([T, \infty) \times \Omega_{+}\right) \cap C^{\infty}\left([T, \infty) \times \Omega_{+}\right)$, multiply both sides of (3.6) by the test function $\phi_{+}$, integrate on $[T, \infty) \times(0, \pi)$, and find

$$
\begin{aligned}
\int_{T}^{\infty} \int_{0}^{\pi} q_{+} \omega_{n_{k},+} \frac{\partial \phi_{+}}{\partial t} \mathrm{~d} \theta \mathrm{d} t & =D_{\theta} \int_{T}^{\infty} \int_{0}^{\pi} \frac{\partial \omega_{n_{k},+}}{\partial \theta} \frac{\partial\left(q_{+} \phi_{+}\right)}{\partial \theta} \mathrm{d} \theta \mathrm{d} t-2 D_{\theta} \int_{T}^{\infty} \int_{0}^{\pi} \frac{\partial q_{+}}{\partial \theta} \frac{\partial \omega_{n_{k},+}}{\partial \theta} \phi_{+} \mathrm{d} \theta \mathrm{d} t \\
& +D_{\theta} \int_{T}^{\infty} \int_{0}^{\pi}\left(\omega_{n_{k},+L}-\omega_{n_{k},+}\right) \frac{\partial^{2} q_{+}}{\partial \theta^{2}} \phi_{+} \mathrm{d} \theta \mathrm{d} t .
\end{aligned}
$$

Let $n_{k} \rightarrow \infty$ and pass to limit, from (3.5), the right hand side of (3.7) vanishes and hence, for any compactly supported $\phi_{+}(t, \theta) \in L^{1}\left([T, \infty) \times \Omega_{+}\right) \cap C^{\infty}\left([T, \infty) \times \Omega_{+}\right)$,

$$
\int_{T}^{\infty} \int_{0}^{\pi} q_{+} \frac{\partial \omega_{\infty,+}}{\partial t} \phi_{+} \mathrm{d} \theta \mathrm{d} t=-\int_{T}^{\infty} \int_{0}^{\pi} q_{+} \omega_{\infty,+} \frac{\partial \phi_{+}}{\partial t} \mathrm{~d} \theta \mathrm{d} t=0 .
$$

Therefore, $\omega_{\infty,+}$ is independent of $t$. Similarly, $\omega_{\infty,-}$ is independent of $t$ as well.

Third step: $\omega_{\infty}$ is independent of $t$ and $y$. Since $q$ is independent of time, from (2.13), for all $k \in \mathbb{N}$, we have

$$
q \frac{\partial \omega_{n_{k}}}{\partial t}=2 D_{\theta} \frac{\partial q}{\partial \theta} \frac{\partial \omega_{n_{k}}}{\partial \theta}+D_{\theta} \frac{\partial^{2} \omega_{n_{k}}}{\partial \theta^{2}} q-V \sin \theta q \frac{\partial \omega_{n_{k}}}{\partial y} .
$$

Letting $n_{k} \rightarrow \infty$ in (3.9) and using (3.5a), gives

$$
q \frac{\partial \omega_{\infty}}{\partial t}=-V \sin \theta q \frac{\partial \omega_{\infty}}{\partial y}
$$

Thanks to the strictly positivity of $q$ inside $\Omega$, we divide both sides of the above equation by $q$, multiply it by a test function $\phi(t, y) \in C^{2}([0, \infty) \times(-L, L))$ and find

$$
\int_{0}^{\infty} \int_{-L}^{L} \phi \frac{\partial \omega_{\infty}}{\partial t} \mathrm{~d} y \mathrm{~d} t=-V \sin \theta \int_{0}^{\infty} \int_{-L}^{L} \phi \frac{\partial \omega_{\infty}}{\partial y} \mathrm{~d} y \mathrm{~d} t
$$

The right hand side of (3.10) depends on $\theta$ while the left hand side does not, which indicates that both sides are zero. Therefore, $\omega_{\infty}$ is independent of $t$ and $y$. So far we proved $\omega_{\infty}$ and $\omega_{\infty, \pm}$ are both constant almost everywhere in their domain of definition respectively.

Forth step: show that $\omega_{\infty}=\omega_{\infty, \pm}$. Multiplying (3.9) by a test function $\phi \in C^{2}([0,+\infty) \times \Omega)$ that is compact supported in $t$ and $\theta$ but not in $y$ and integrating on $[T, \infty) \times \Omega$ yields

$$
\begin{aligned}
\int_{T}^{\infty} \int_{-L}^{L} \int_{-\pi}^{\pi} & q \omega_{n_{k}} \frac{\partial \phi}{\partial t} \mathrm{~d} \theta \mathrm{d} y \mathrm{~d} t=\int_{T}^{\infty} \int_{-\pi}^{\pi} V \sin \theta \int_{-L}^{L} q \phi \frac{\partial \omega_{n_{k}}}{\partial y} \mathrm{~d} y \mathrm{~d} \theta \mathrm{d} t \\
& +D_{\theta} \int_{T}^{\infty} \int_{-L}^{L} \int_{-\pi}^{\pi} \frac{\partial \omega_{n_{k}}}{\partial \theta} \frac{\partial(q \phi)}{\partial \theta} \mathrm{d} \theta \mathrm{d} y \mathrm{~d} t-2 D_{\theta} \int_{T}^{\infty} \int_{-L}^{L} \int_{-\pi}^{\pi} \frac{\partial q}{\partial \theta} \frac{\partial \omega_{n_{k}}}{\partial \theta} \phi \mathrm{d} \theta \mathrm{d} y \mathrm{~d} t \\
& +\left.D_{\theta} \int_{T}^{\infty}\left[\left(\omega_{n_{k},+}-\omega_{n_{k}, L}\right) \frac{\partial q_{+}}{\partial \theta}\right] \phi(t, L, \theta)\right|_{0} ^{\pi} \mathrm{d} t+\left.D_{\theta} \int_{T}^{\infty}\left[\left(\omega_{n_{k},-}-\omega_{n_{k},-L}\right) \frac{\partial q_{-}}{\partial \theta}\right] \phi(t,-L, \theta)\right|_{-\pi} ^{0} \mathrm{~d} t
\end{aligned}
$$


Here the last term on the right hand side comes from the boundary conditions for $p$ and $q$ in (1.5), (1.6) and (2.2). Besides, using integration by part, we find

$$
\begin{aligned}
& \int_{T}^{\infty} \int_{-\pi}^{\pi} V \sin \theta \int_{-L}^{L} q \phi \frac{\partial \omega_{n_{k}}}{\partial y} \mathrm{~d} y \mathrm{~d} \theta \mathrm{d} t \\
= & -\int_{T}^{\infty} \int_{-\pi}^{\pi} V \sin \theta \int_{-L}^{L} \omega_{n_{k}} \frac{\partial(q \phi)}{\partial y} \mathrm{~d} y \mathrm{~d} \theta \mathrm{d} t+\left.\int_{T}^{\infty} \int_{-\pi}^{\pi} V \sin \theta\left(\phi q \omega_{n_{k}}\right)\right|_{-L} ^{L} \mathrm{~d} \theta \mathrm{d} t \\
= & -\int_{T}^{\infty} \int_{-\pi}^{\pi} V \sin \theta \int_{-L}^{L} \omega_{n_{k}} \frac{\partial(q \phi)}{\partial y} \mathrm{~d} y \mathrm{~d} \theta \mathrm{d} t+\int_{T}^{\infty} \int_{0}^{\pi} V \sin \theta \phi_{L} \omega_{n_{k},+} q_{+L} \mathrm{~d} \theta \mathrm{d} t-\int_{T}^{\infty} \int_{-\pi}^{0} V \sin \theta \phi_{-L} \omega_{n_{k},-} q_{-L} \mathrm{~d} \theta \mathrm{d} t \\
& -\int_{T}^{\infty} \int_{0}^{\pi} V \sin \theta \phi_{L}\left(\omega_{n_{k},+}-\omega_{n_{k}, L}\right) q_{+L} \mathrm{~d} \theta \mathrm{d} t+\int_{T}^{\infty} \int_{-\pi}^{0} V \sin \theta \phi_{-L}\left(\omega_{n_{k},-}-\omega_{n_{k},-L}\right) q_{-L} \mathrm{~d} \theta \mathrm{d} t .
\end{aligned}
$$

Therefore using (3.5), when $n_{k} \rightarrow \infty$,

$$
\begin{aligned}
& \int_{T}^{\infty} \int_{-L}^{L} \int_{-\pi}^{\pi} \omega_{\infty} \frac{\partial(q \phi)}{\partial t} \mathrm{~d} \theta \mathrm{d} y \mathrm{~d} t \\
= & -\int_{T}^{\infty} \int_{-L}^{L} \int_{-\pi}^{\pi} \omega_{\infty} V \sin \theta \frac{\partial(q \phi)}{\partial y} \mathrm{~d} \theta \mathrm{d} y \mathrm{~d} t+\int_{T}^{\infty} \int_{0}^{\pi} V \sin \theta \phi_{L} \omega_{\infty,+} q_{+L} \mathrm{~d} \theta \mathrm{d} t \\
& -\int_{T}^{\infty} \int_{-\pi}^{0} V \sin \theta \phi_{-L} \omega_{\infty,-} q_{-L} \mathrm{~d} \theta \mathrm{d} t \\
= & -\left(\omega_{\infty}-\omega_{\infty,+}\right) \int_{T}^{\infty} \int_{-L}^{L} \int_{0}^{\pi} V \sin \theta \frac{\partial(q \phi)}{\partial y} \mathrm{~d} \theta \mathrm{d} y \mathrm{~d} t-\left(\omega_{\infty}-\omega_{\infty,-}\right) \int_{T}^{\infty} \int_{-L}^{L} \int_{-\pi}^{0} V \sin \theta \frac{\partial(q \phi)}{\partial y} \mathrm{~d} \theta \mathrm{d} y \mathrm{~d} t .
\end{aligned}
$$

Here the last equation has used the second boundary condition in (2.2).

We can choose $\phi(t, y, \theta)$ that is compactly supported in $(0,+\infty)$ for $t$ and $(0, \pi)$ for $\theta$ and satisfies

$$
\int_{T}^{\infty} \int_{-L}^{L} \int_{0}^{\pi} V \sin \theta \frac{\partial(q \phi)}{\partial y} \mathrm{~d} \theta \mathrm{d} y \mathrm{~d} t=\left.\int_{T}^{\infty} \int_{0}^{\pi} V \sin \theta(q \phi)\right|_{-L} ^{L} \mathrm{~d} \theta \mathrm{d} y \mathrm{~d} t \neq 0 .
$$

Then (3.12) indicates that $\omega_{\infty}=\omega_{\infty,+}=C$. Similarly, we have $\omega_{\infty}=\omega_{\infty,-}$. Thus there exist a constant $C$ such that

$$
\left.\omega_{\infty}\right|_{\Omega}=\left.\omega_{\infty, \pm}\right|_{\Omega_{ \pm}}=C, \quad \text { a.e. }
$$

By the conservation of mass, $\forall n_{k} \in \mathbb{N}, \omega_{n_{k}}$ satisfies

$$
\int_{-L}^{L} \int_{-\pi}^{\pi} q \omega_{n_{k}} \mathrm{~d} \theta \mathrm{d} y+\int_{0}^{\pi} q_{+} \omega_{n_{k},+} \mathrm{d} \theta+\int_{-\pi}^{0} q_{-} \omega_{n_{k},-} \mathrm{d} \theta=0,
$$

which indicates that

$$
\int_{-L}^{L} \int_{-\pi}^{\pi} q \omega_{\infty} \mathrm{d} \theta \mathrm{d} y+\int_{0}^{\pi} q_{+} \omega_{\infty,+} \mathrm{d} \theta+\int_{-\pi}^{0} q_{-} \omega_{\infty,-} \mathrm{d} \theta=0 .
$$

Therefore, the constant $C$ has to be zero.

Fifth step: strong convergence in time. For a smooth test function $\phi(y, \theta) \in C_{0}^{2}(\Omega)$, we define

$$
u(t)=\int_{\Omega} q \phi \omega \mathrm{d} y \mathrm{~d} \theta .
$$


Multiplying both sides of (2.13) by $\phi(y, \theta)$ and integrating over $\Omega$ gives

$$
\int_{\Omega} \frac{\partial(q \phi \omega)}{\partial t} \mathrm{~d} y \mathrm{~d} \theta=\int_{\Omega} \phi \frac{\partial p}{\partial t} \mathrm{~d} y \mathrm{~d} \theta=\int_{\Omega} \phi\left(D_{\theta} \frac{\partial^{2} p}{\partial \theta^{2}}-V \sin \theta \frac{\partial p}{\partial y}\right) \mathrm{d} y \mathrm{~d} \theta<C^{\prime}
$$

Here $C^{\prime}$ in the last inequality is some positive constant and the inequality is easy to prove by using integral by parts and Corollary 2.2. Therefore, $u(t)$ is a Lipschitz function in time and we have shown that it converges weakly to 0 , then it converges strongly. Strong convergence of $\omega_{ \pm}$in time can be derived similarly.

\section{$4 \quad$ Numerical simulations}

The steady state problem (2.1) and (2.2) is a linear eigenvalue problem and only unique under the constraint (2.3). We can simulate it by fully implicit scheme based on an entropy decreasing semi-discrete scheme for the time evolution problem (1.1). The discrete entropy estimate of the semi-discrete scheme is established in the Appendix and we observe the convergence to steady state numerically. More sophisticated methods, with exponential decay, have been proposed, not including the difficulty related to the boundary, see [10] and the references therein.

Dimensionless parameters are used in our numerical solver and simulations. Rotational diffusion coefficient is $D_{\theta}=1$, moving speed is $V=20$, and distance between the two horizontal plates are $2 L=20$.

\subsection{Description of the finite difference scheme}

We consider a uniform mesh for $y$ and $\theta$ in the rectangular domain $\Omega=[-L, L] \times[-\pi, \pi]$. Let the mesh sizes of $y$ and $\theta$ be respectively $\Delta y=L / I$ and $\Delta \theta=\pi / J$ where $I, J$ are two integers. The index sets are

$$
V_{y}=\{1,2, \cdots, 2 I\}, \quad V_{\theta}=\{1,2, \cdots, 2 J\}, \quad \tilde{V}_{\theta}=\{0,1, \cdots, 2 J\},
$$

and

$$
V_{\theta_{+}}=\{J+1, J+2, \cdots, 2 J-1\}, \quad V_{\theta_{-}}=\{1,2, \cdots, J-1\}, \quad V_{\theta_{0}}=\{0, J, 2 J\} .
$$

As in Figure 1, the grids inside the computational domain are:

$$
\left(y_{i}, \theta_{j}\right)=((i-I-1 / 2) \Delta y,(j-J) \Delta \theta), \quad i \in V_{y}, \quad j \in \tilde{V}_{\theta}
$$

and the nodes at the boundaries are:

$$
\left( \pm L, \theta_{j}\right), \quad j \in V_{\theta_{ \pm}} \bigcup V_{\theta_{0 \pm}}
$$

The unknowns are $p_{ \pm, j} \approx p_{ \pm}\left(t, \theta_{j}\right)$ and

$$
p_{i, j} \approx \frac{1}{\Delta y} \int_{y_{i}-\Delta y / 2}^{y_{i}+\Delta y / 2} p\left(t, y_{i}, \theta_{j}\right) \mathrm{d} y
$$

We use upwind discretization for $\partial_{y} p$, central finite difference discretization for $\frac{\partial^{2} p}{\partial \theta^{2}}$, and the discretizations of 


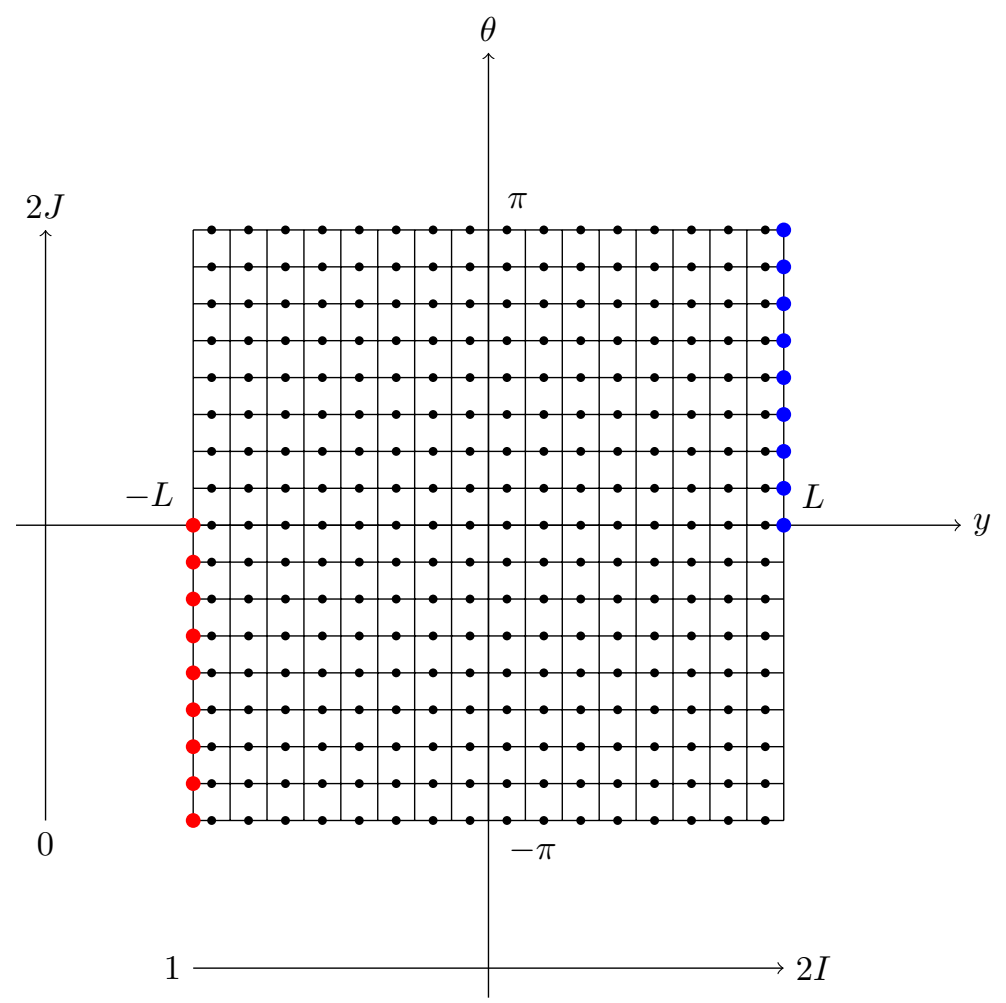

Figure 1: Stencil of semi-discretized scheme. Here nodes for $p\left(t, y_{i}, \theta_{j}\right)$ inside $\Omega$ are black, while nodes for $p_{ \pm}$are blue and red respectively.

boundary conditions are chosen to preserve the total mass. The semi-discretized scheme writes

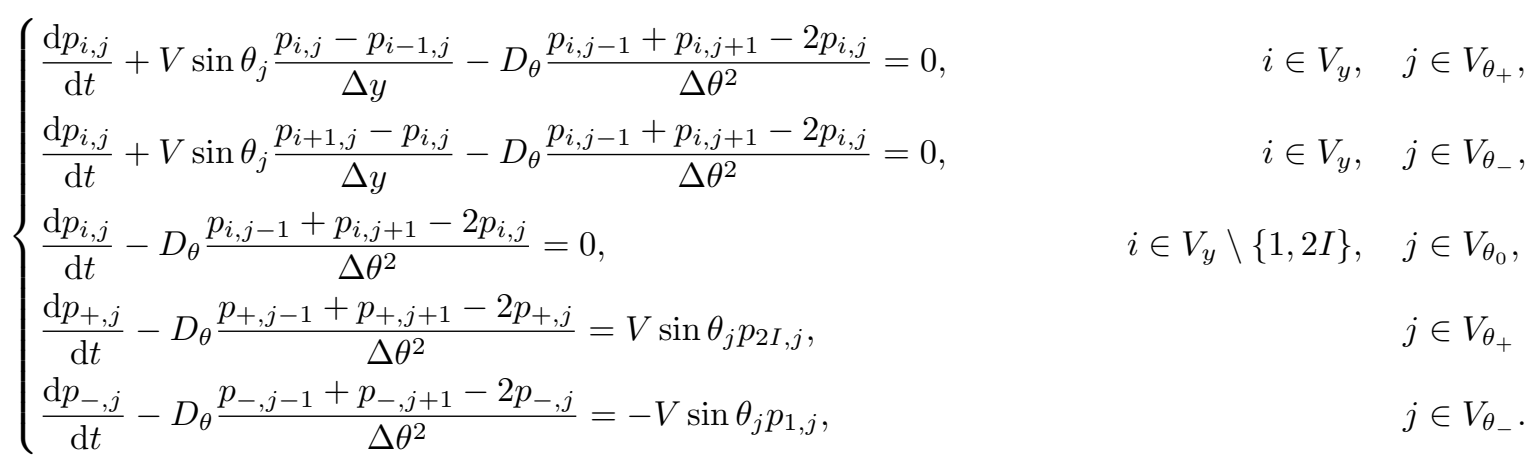

From the periodic boundary conditions in (1.2) for $p(t, y, \theta)$ in $\theta$, we use

$$
p_{i,-1}=p_{i, 2 J-1}, \quad p_{i, 0}=p_{i, 2 J}, \quad p_{i, 2 J+1}=p_{i, 1}, \quad \forall i \in V_{y} ;
$$

and due to the zero boundary conditions in (1.3) and (1.4), we use

$$
p_{0, j}=0, \quad j \in V_{\theta_{+}}, \quad p_{2 I+1, j}=0, \quad j \in V_{\theta_{-}}, \quad p_{+, J}=p_{+, 2 J}=0, \quad p_{-, 0}=p_{-, J}=0 .
$$


At the continuous level, $p$ is integrated over the whole space, the conservation of mass can be obtained because the boundary conditions of $p$ and $p_{ \pm}$cancel each other. On the other hand, at the discrete level, if we replace evolution equations by boundary conditions at the four points $(1, J),(1,0),(2 I, J),(2 I, 2 J)$, then the information about how density function evolves at these point are lost. Hence we prefer to discretize the equation (1.7)-(1.8) mentioned in Remark 1.1, which is equivalent to our model.

Approximate equation at the point $(-L, 0)$ reads

$$
\frac{\mathrm{d} p_{1, J}}{\mathrm{~d} t}-D_{\theta} \frac{p_{1, J-1}+p_{1, J+1}-2 p_{1, J}}{\Delta \theta^{2}}=D_{\theta} \frac{p_{-, J}-p_{-, J-1}}{\Delta \theta^{2} \Delta y} .
$$

Similarly, at $(-L,-\pi),(L, 0),(-L, \pi)$, we have

$$
\begin{gathered}
\frac{\mathrm{d} p_{1,1}}{\mathrm{~d} t}-D_{\theta} \frac{p_{1,-1}+p_{1,1}-2 p_{1,0}}{\Delta \theta^{2}}=-D_{\theta} \frac{p_{-, 1}-p_{-, 0}}{\Delta \theta^{2} \Delta y}, \\
\frac{\mathrm{d} p_{2 I, J}}{\mathrm{~d} t}-D_{\theta} \frac{p_{2 I, J-1}+p_{2 I, J+1}-2 p_{2 I, J}}{\Delta \theta^{2}}=-D_{\theta} \frac{p_{+, J+1}-p_{+, J}}{\Delta \theta^{2} \Delta y}, \\
\frac{\mathrm{d} p_{2 I, 2 J}}{\mathrm{~d} t}-D_{\theta} \frac{p_{2 I, 2 J+1}+p_{2 I, 2 J-1}-2 p_{2 I, 2 J}}{\Delta \theta^{2}}=D_{\theta} \frac{p_{+, 2 J}-p_{+, 2 J-1}}{\Delta \theta^{2} \Delta y} .
\end{gathered}
$$

Non-negative initial data $p_{i, j}(0)$ and $p_{ \pm, j}(0)$ that satisfy

$$
\Delta \theta \Delta y \sum_{(i, j) \in V_{y} \times V_{\theta}} p_{i, j}(0)+\Delta \theta \sum_{j \in V_{\theta_{+}}} p_{+, j}(0)+\Delta \theta \sum_{j \in V_{\theta_{-}}} p_{-, j}(0)=1 .
$$

is used and it is easy to verify that the scheme (4.2)-(4.4) conserves the discretized total mass defined by

$$
\Delta \theta \Delta y \sum_{(i, j) \in V_{y} \times V_{\theta}} p_{i, j}(t)+\Delta \theta \sum_{j \in V_{\theta_{+}}} p_{+, j}(t)+\Delta \theta \sum_{j \in V_{\theta_{-}}} p_{-, j}(t)
$$

In Appendix, we proved the discrete relative entropy estimate for (4.2)-(4.5). In the subsequent part, we can see numerically that when $t \rightarrow \infty$, the solution converges to the solution of the following discretized steady state equation:

$$
\left\{\begin{array}{lr}
V \sin \theta_{j} \frac{q_{i, j}-q_{i-1, j}}{\Delta y}-D_{\theta} \frac{q_{i, j-1}+q_{i, j+1}-2 q_{i, j}}{\Delta \theta^{2}}=0, & i \in V_{y}, \quad j \in V_{\theta_{+}}, \\
V \sin \theta_{j} \frac{q_{i+1, j}-q_{i, j}}{\Delta y}-D_{\theta} \frac{q_{i, j-1}+q_{i, j+1}-2 q_{i, j}}{\Delta \theta^{2}}=0, & i \in V_{y}, \quad j \in V_{\theta_{-}}, \\
-D_{\theta} \frac{q_{i, j-1}+q_{i, j+1}-2 q_{i, j}}{\Delta \theta^{2}}=0, & i \in V_{y} \backslash\{1,2 I\}, \quad j \in V_{\theta_{0+}} \cup V_{\theta_{0-}}, \\
-D_{\theta} \frac{q_{+, j-1}+q_{+, j+1}-2 q_{+, j}}{\Delta \theta^{2}}=V \sin \theta_{j} q_{2 I, j}, & j \in V_{\theta_{+}} \\
-D_{\theta} \frac{q_{-, j-1}+q_{-, j+1}-2 q_{-, j}}{\Delta \theta^{2}}=-V \sin \theta_{j} q_{1, j}, & j \in V_{\theta_{-}} .
\end{array}\right.
$$


with boundary conditions

$$
\begin{aligned}
& q_{i,-1}=q_{i, 2 J-1}, q_{i, 0}=q_{i, 2 J}, q_{i, 1}=q_{i, 2 J+1}, \quad i \in V_{y} \\
& q_{0, j}=0, \quad j \in V_{\theta_{+}}, \quad q_{2 I+1, j}=0 ; \quad j \in V_{\theta_{-}} \\
& q_{+, J}=q_{+, 2 J}=0 ; \quad q_{-, 0}=q_{-, J}=0 ; \\
& q_{1,-1}+q_{1,1}-2 q_{1,0}+\frac{q_{-, 1}}{\Delta y}=0, \quad q_{1, J-1}+q_{1, J+1}-2 q_{1, J}+\frac{q_{-, J-1}}{\Delta y}=0, \\
& q_{2 I, J-1}+q_{2 I, J+1}-2 q_{2 I, J}+\frac{q_{+, J+1}}{\Delta y}=0, \quad q_{2 I, 2 J-1}+q_{2 I, 2 J+1}-2 q_{2 I, 2 J}+\frac{q_{+, 2 J-1}}{\Delta y}=0,
\end{aligned}
$$

and total mass normalization

$$
\Delta \theta \Delta y \sum_{(i, j) \in V_{y} \times V_{\theta}} q_{i, j}+\Delta \theta \sum_{j \in V_{\theta_{+}}} q_{+, j}+\Delta \theta \sum_{j \in V_{\theta_{-}}} q_{-, j}=1 .
$$

We have used upwind discretization for $\partial_{y} p$, which has first order convergence w.r.t $y$; second order centered finite difference discretization is employed for $\frac{\partial^{2} p}{\partial \theta^{2}}$, but due to first order discretization for derivatives in the source term (1.8) as in (4.4), the scheme has first order convergence w.r.t $\theta$. We define the following weighted $L^{2}$ norm to measure the numerical errors:

$$
\left\|\left(e, e_{+}, e_{-}\right)\right\|_{L_{w}^{2}}^{2}=\sum_{i \in V_{y}} \sum_{j \in V_{\theta}} \Delta \theta^{2} \Delta y^{2} e_{i, j}^{2}+\sum_{j \in V_{\theta_{+}}} \Delta \theta^{2} e_{+, j}^{2}+\sum_{j \in V_{\theta_{-}}} \Delta \theta^{2} e_{-, j}^{2} .
$$

In Table 1, numerical errors of $q_{i, j}$ calculated with different mesh sizes are given, where the reference solution is computed with a fine mesh $256 \times 256$. From Figure 2, first order convergence can be observed when $I=J$.

\begin{tabular}{|l|c|c|c|c|}
\hline$\Delta y$ & $\pi / 128$ & $\pi / 64$ & $\pi / 32$ & $\pi / 16$ \\
\hline$L / 128$ & $5.2 \mathrm{E}-4$ & $7.3 \mathrm{E}-4$ & $9.4 \mathrm{E}-4$ & $1.5 \mathrm{E}-3$ \\
\hline$L / 64$ & $1.1 \mathrm{E}-3$ & $1.6 \mathrm{E}-3$ & $2.2 \mathrm{E}-3$ & $2.5 \mathrm{E}-3$ \\
\hline$L / 32$ & $1.9 \mathrm{E}-3$ & $2.7 \mathrm{E}-3$ & $3.8 \mathrm{E}-3$ & $4.9 \mathrm{E}-3$ \\
\hline$L / 16$ & $2.9 \mathrm{E}-3$ & $4.1 \mathrm{E}-3$ & $5.7 \mathrm{E}-3$ & $7.7 \mathrm{E}-3$ \\
\hline
\end{tabular}

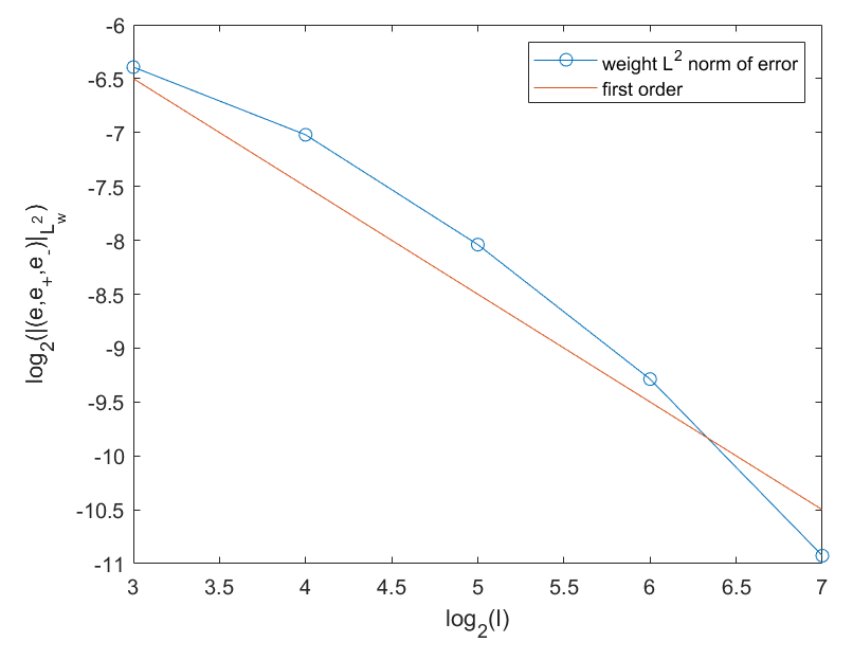

Table 1: $\left\|\left(q, q_{+}, q_{-}\right)-\left(q^{r e f}, q_{+}^{r e f}, q_{-}^{r e f}\right)\right\|_{L_{w}^{2}}$. The weighted $L^{2}$ norm of the numerical errors as in (4.9)

Figure 2: The convergence order of the numerical errors displayed in Table 1 when $I=J$. 


\subsection{Numerical comparisons between the SDE and Fokker-Planck models}

We perform Monte-Carlo simulations for the SDE model in (0.1) with Euler-Maruyama-like scheme [17]. The computational domain is $\bar{\Omega}=\{(y, \theta) \mid-L \leq y \leq L,-\pi \leq \theta \leq \pi\}$ and we track the trajectories of $K=5000000$ cells. Each cell is represented by its position $y^{i}$, orientation $\theta^{i}$. The initial $y^{i}$ for all cells are uniformly distributed on $(-L, L)$, their initial orientation $\theta^{i}$ are uniformly distributed on $[-\pi, \pi]$. Let $\Delta t$ be the time step. At each step we evolve $\left(y^{i}, \theta^{i}\right)$ $(i=1, \cdots, K)$ by the following calculations:

1) Update $\theta^{i}$ by $\mathrm{d} \Theta_{t}=\sqrt{2 D_{\theta}} \mathrm{d} W_{t}$. For each $i$, generate a random increment $I^{i}$ with normal distribution independent of previous ones. Suppose that $\theta^{i}+\sqrt{2 D_{\theta} \Delta t} I^{i} \in[2 k \pi-\pi, 2 k \pi+\pi)$ for some integer $\mathrm{k}$, then set the new orientation $\theta^{i}$ to be $\theta^{i}+\sqrt{2 D_{\theta} \Delta t} I^{i}-2 k \pi$.

2) Update the position $y^{i}$. If $y^{i}= \pm L$, and $\pm \sin \left(\theta^{i+1}\right) \geq 0$, then $y^{i}$ does not change. If $\left|y^{i}\right|<L,\left|y^{i}+V \sin \left(\theta^{i+1}\right)\right|>L$, then the new $y^{i}$ is set to be $\operatorname{sign}\left(y^{i}+V \sin \left(\theta^{i}\right)\right) L$. Otherwise, evolve $y^{i}$ by $y^{i}+V \sin \left(\theta^{i+1}\right)$.

We take $\Delta t=0.01$ and count the number of particles inside each cell of a $200 \times 200$ uniform mesh and normalize them by the total number of particles. It has been found in simulations that, the probability distribution is close to steady state when $T=4$.

Finite difference scheme described in section 4.1 is used to compute the numerical solution of Fokker-Planck model. In practice, we take $I=J=100$ such that the numerical results of two model have same resolution. Fully implicit scheme is performed on the semi-discretized equation (4.2), and we employed GMRES to solve the resulting algebraic system. The initial condition is consistent with SDE model, i.e. $p_{i, j}^{0}=\frac{1}{4 \pi L}$ for all $i \in V_{y}, j \in V_{\theta}$. The same as the SDE model, solution of the Fokker-Planck equation is close to steady state when $T=4$.

The time evolutions of bulk density $p(t, y, \theta)$ by numerical simulations based on both SDE and Fokker-Planck models are shown in Figure 3. The results of the two models are close to each other qualitatively. There exist two wells when $y=-L, \theta \in(0, \pi)(y=L, \theta \in(-\pi, 0))$, since cells with positive (negative) $\theta$ tend to leave the boundary. When $t$ is larger, the density peaks at $( \pm L, 0),( \pm L, \pm \pi)$, since cells switch from BCP to FSP only at these points. We can see density in most of bulk area is uniform, and decreases as $t$ increases.

To verify the behavior of $p(t, y, \theta)$ near the boundaries, $p_{1, j}$ and $p_{2 I, j}$ are plotted in Figure 4 . As $t$ becomes larger, the peaks at $( \pm L, 0),( \pm L, \pm \pi)$ become sharper. Due to the limitation of numerical resolutions, the height of peaks stop increasing when $t$ reaches 4 . It is not clear from the numerics if there exist singularities in these points. The probability density distributions of cells at BCP on both left and right boundaries are plotted in Figure 4 as well, we can see that their maximum increase with time until they reach a constant.

\section{Conclusion}

Motivated by present biophysics experiments, we have considered the stochastic process which describes self-propelled confined micro-organisms moving with constant speed, turning with rotational Brownian motion and attaching to the boundaries. Its probability distribution is singular and composed of the bulk density $p(t, y, \theta)$ and boundary densities $p_{ \pm}(t, \theta)$. These functions satisfy a degenerate Fokker-Planck system for which we have established relative entropy properties, a priori estimates and long term convergence to a steady-state. We have finally compared Monte-Carlo simulations for the stochastic process with the solution of the Fokker-Planck system using a finite difference scheme. Despite the complexity and singularities of the solution, a good fit is observed.

Several questions are left opened as the regularity properties of the time dependent solution and of the steady state solution. In particular, the type of singularity at the exit points of the boundary is challenging, It would be also interesting to prove a rate of convergence to steady state and decide it is exponential or not. There are also several 

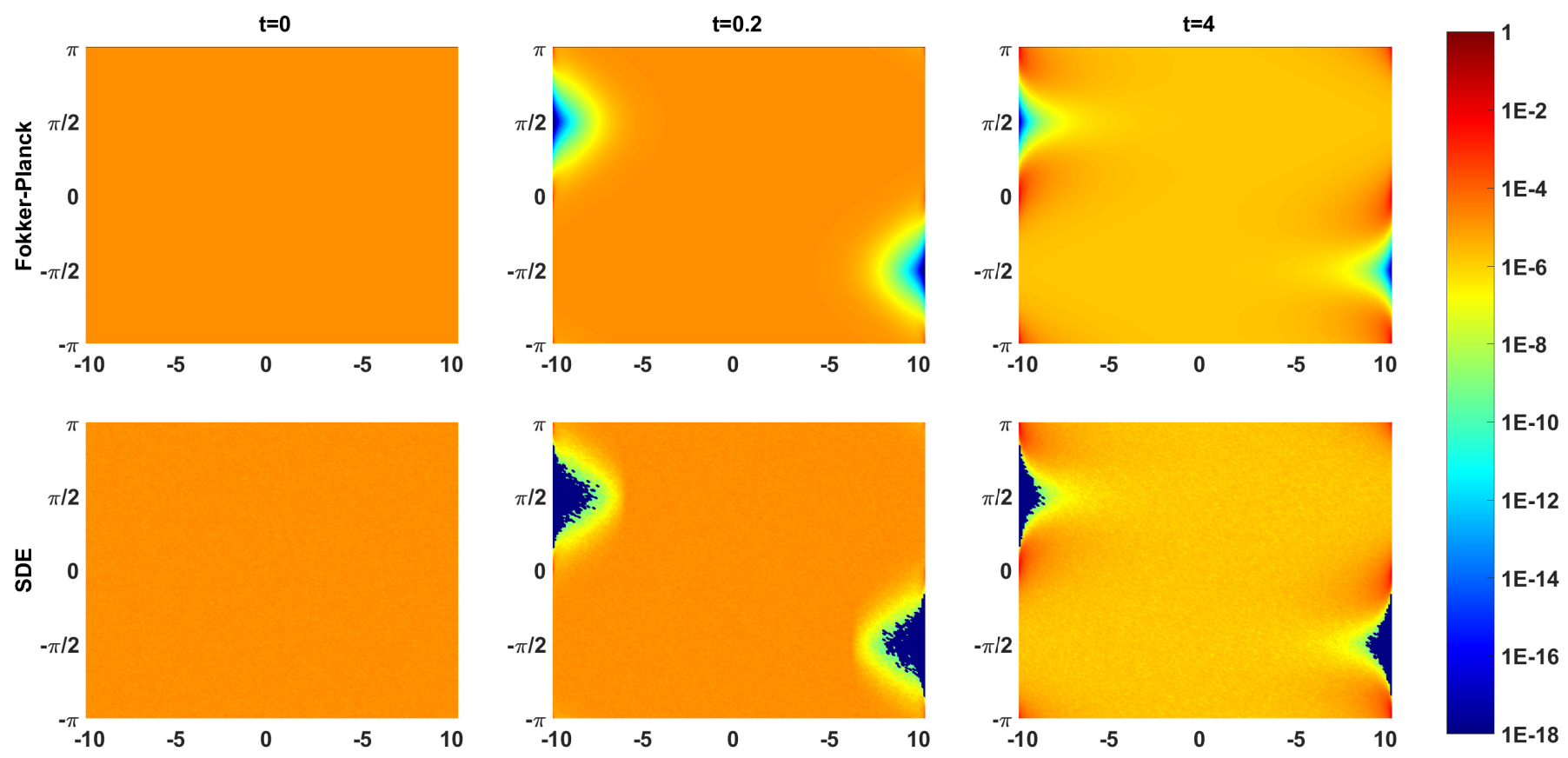

Figure 3: Density in the bulk $p_{i, j}$ at different time calculated by Fokker Plank model and SDE model.

open questions on the numerical side, for instance the proof for convergence of the scheme and the design of numerical schemes with good decay properties.

\section{A Discrete relative entropy inequality and a priori estimate}

The semi-discrete relative gap $\omega_{i, j}(t)$ and $\omega_{ \pm, j}(t)$ are defined by

$$
\left\{\begin{array}{lll}
\omega_{i, j}(t)=\frac{p_{i, j}(t)}{q_{i, j}}-1, & i \in V_{y} \backslash\{1\}, & j \in V_{\theta_{+}}, \\
\omega_{1, j}(t)=0, \quad \omega_{+, j}(t)=\frac{p_{+, j}(t)}{q_{+, j}}-1, & i \in V_{y} \backslash\{2 I\}, & j \in V_{\theta_{-}}, \\
\omega_{2 I, j}(t)=0, \quad \omega_{-, j}(t)=\frac{p_{-, j}(t)}{q_{-, j}}-1, & j \in V_{\theta_{+}}, \\
& j \in V_{\theta_{-}},
\end{array}\right.
$$

Here we state the discrete relative entropy inequality which is satisfied by discrete density $p_{i, j}(t)$ and $p_{ \pm, j}(t)$.

Theorem A.1. For any convex function $H \in \mathcal{C}^{2}(\mathbb{R})$, semi-discrete relative gap $\omega_{i, j}(t)$ and $\omega_{ \pm, j}(t)$ satisfy the relative entropy inequality:

$$
\frac{\mathrm{d}}{\mathrm{d} t}\left[\sum_{i \in V_{y}} \sum_{j \in V_{\theta}} q_{i, j} H\left(\omega_{i, j}\right)+\sum_{j \in V_{\theta_{+}}} q_{+, j} H\left(\omega_{+, j}\right)+\sum_{j \in V_{\theta_{-}}} q_{-, j} H\left(\omega_{-, j}\right)\right] \leq 0,
$$


$t=0$
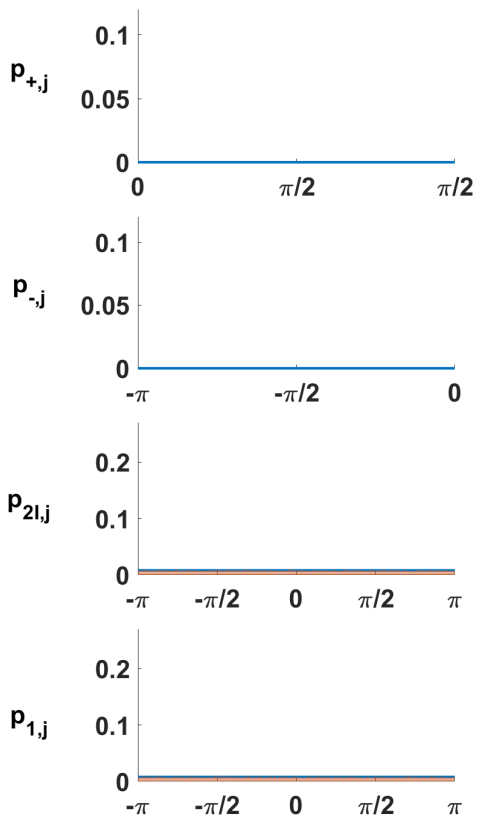

$\mathrm{t}=0.2$
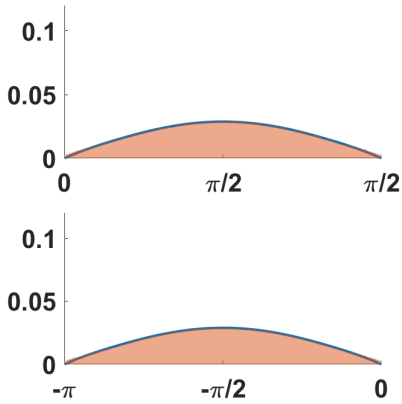

0.2

0.1
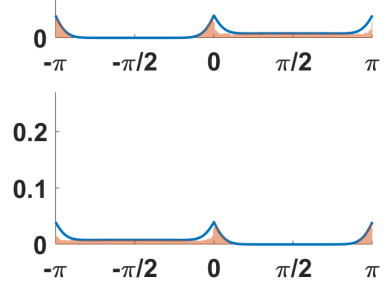

$t=0.5$
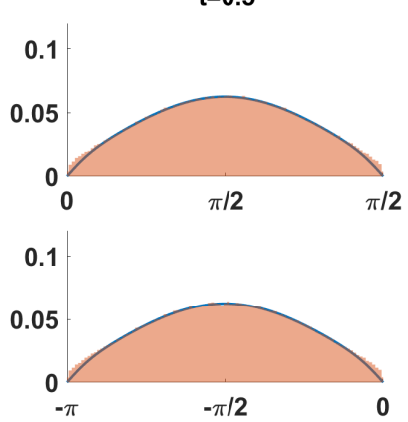

0.2
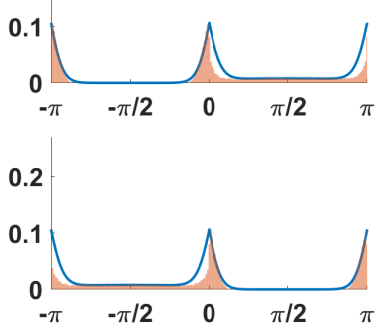
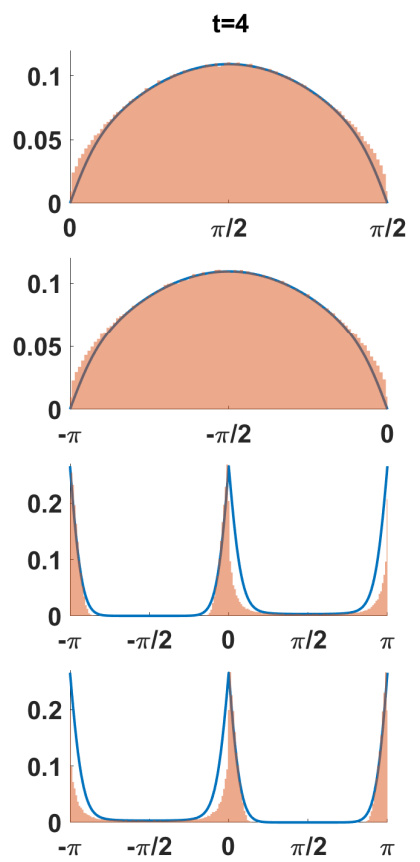

Figure 4: Density in the bulk near boundary $p_{1, j}, p_{2 I, j}$ and boundary density $p_{-, j}$ and $p_{+, j}$ (from top to bottom) at different times calculated by the Fokker Plank model (line) and SDE model (histogram). Notice that $p_{1, j}$ is close to $\{y=-L\}$ but not at the boundary, so the boundary condition (1.3) is not satisfied for $p_{1, j}$.

where equality holds if and only if $\omega_{i, j}=0$ for any $i \in V_{y}, j \in V_{\theta}$ and $\omega_{ \pm, j}=0$ for any $j \in V_{\theta, \pm}$.

Proof. As in section 2, we divide the proof in several steps.

First step: estimate of relative entropy at BCP. Substitute $p_{+, j}$ by $q_{+, j} \omega_{+, j}+q_{+, j}$ in $(4.2 \mathrm{~d})$ and employ (4.6d), we deduce that

$$
\begin{aligned}
q_{+, j} \frac{\mathrm{d} \omega_{+, j}}{\mathrm{~d} t}= & \frac{D_{\theta}}{\Delta \theta^{2}}\left[\left(q_{+, j-1} \omega_{+, j-1}+q_{+, j+1} \omega_{+, j+1}-2 q_{+, j} \omega_{+, j}\right)+\left(q_{+, j-1}+q_{+, j+1}-2 q_{+, j}\right)\right] \\
& +V \sin \theta_{j} q_{2 I, j}\left(\omega_{2 I, j}+1\right) \\
= & \frac{D_{\theta}}{\Delta \theta^{2}}\left[\left(q_{+, j-1} \omega_{+, j-1}+q_{+, j+1} \omega_{+, j+1}-2 q_{+, j} \omega_{+, j}\right)-\omega_{2 I, j}\left(q_{+, j-1}+q_{+, j+1}-2 q_{+, j}\right)\right] \\
= & \frac{D_{\theta}}{\Delta \theta^{2}}\left[q_{+, j+1}\left(\omega_{+, j+1}-\omega_{+, j}\right)-q_{+, j-1}\left(\omega_{+, j}-\omega_{+, j-1}\right)+\left(\omega_{+, j}-\omega_{2 I, j}\right)\left(q_{+, j-1}+q_{+, j+1}-2 q_{+, j}\right)\right] .
\end{aligned}
$$

Notice that

$$
\begin{aligned}
& q_{+, j-1} H\left(\omega_{+, j-1}\right)+q_{+, j+1} H\left(\omega_{+, j+1}\right)-2 q_{+, j} H\left(\omega_{+, j}\right) \\
= & q_{+, j+1}\left[H\left(\omega_{+, j+1}\right)-H\left(\omega_{+, j}\right)\right]-q_{+, j-1}\left[H\left(\omega_{+, j}\right)-H\left(\omega_{+, j-1}\right)\right]+H\left(\omega_{+, j}\right)\left(q_{+, j-1}+q_{+, j+1}-2 q_{+, j}\right),
\end{aligned}
$$


by rearranging $H^{\prime}\left(\omega_{+, j}\right)(\mathrm{A} .3)-\frac{D_{\theta}}{\Delta \theta^{2}}(\mathrm{~A} .4)$, it follows that

$$
\begin{aligned}
& q_{+, j} \frac{\mathrm{d} H\left(\omega_{+, j}\right)}{\mathrm{d} t}-\frac{D_{\theta}}{\Delta \theta^{2}}\left[q_{+, j-1} H\left(\omega_{+, j-1}\right)+q_{+, j+1} H\left(\omega_{+, j+1}\right)-2 q_{+, j} H\left(\omega_{+, j}\right)\right] \\
= & -\frac{D_{\theta}}{\Delta \theta^{2}} q_{+, j+1}\left[H\left(\omega_{+, j+1}\right)-H\left(\omega_{+, j}\right)-H^{\prime}\left(\omega_{+, j}\right)\left(\omega_{+, j+1}-\omega_{+, j}\right)\right] \\
& -\frac{D_{\theta}}{\Delta \theta^{2}} q_{+, j-1}\left[H^{\prime}\left(\omega_{+, j}\right)\left(\omega_{+, j}-\omega_{+, j-1}\right)-H\left(\omega_{+, j}\right)+H\left(\omega_{+, j-1}\right)\right] \\
& -\left[H^{\prime}\left(\omega_{+, j}\right)\left(\omega_{+, j}-\omega_{2 I, j}\right)-H\left(\omega_{+, j}\right)+H\left(\omega_{2 I, j}\right)\right] V \sin \theta_{j} q_{2 I, j}+H\left(\omega_{2 I, j}\right) V \sin \theta_{j} q_{2 I, j}, \\
\leq & H\left(\omega_{2 I, j}\right) V \sin \theta_{j} q_{2 I, j}
\end{aligned}
$$

where equality holds if and only if $\omega_{+, j-1}=\omega_{+, j}=\omega_{+, j+1}=\omega_{2 I, j}$.

Sum up (A.5) for $j \in V_{\theta_{+}}$, we have

$$
\frac{\mathrm{d}}{\mathrm{d} t} \sum_{j \in V_{\theta_{+}}} q_{+, j} H\left(\omega_{+, j}\right)+\frac{D_{\theta}}{\Delta \theta^{2}} q_{+, J+1} H\left(\omega_{+, J+1}\right)+\frac{D_{\theta}}{\Delta \theta^{2}} q_{+, 2 J-1} H\left(\omega_{+, 2 J}\right) \leq \sum_{j \in V_{\theta_{+}}} H\left(\omega_{2 I, j}\right) V \sin \theta_{j} q_{2 I, j},
$$

where equality holds if and only if $\omega_{+, j}=\omega_{2 I, j}=0$ for any $j \in V_{\theta_{+}}$.

Similarly, we obtain an inequality for $q_{-, j} H\left(\omega_{-, j}\right)$

$$
\frac{\mathrm{d}}{\mathrm{d} t} \sum_{j \in V_{\theta_{-}}} q_{-, j} H\left(\omega_{-, j}\right)+\frac{D_{\theta}}{\Delta \theta^{2}} q_{-, 1} H\left(\omega_{-, 1}\right)+\frac{D_{\theta}}{\Delta \theta^{2}} q_{-, J} H\left(\omega_{-, J-1}\right) \leq-\sum_{j \in V_{\theta_{-}}} H\left(\omega_{1, j}\right) V \sin \theta_{j} q_{1, j}
$$

where equality holds if and only if $\omega_{-, j}=\omega_{1, j}=0$ for any $j \in V_{\theta_{-}}$.

Second step: estimate of relative entropy at FSP. Substituting $p_{i, j}$ by $q_{i, j} \omega_{i, j}+q_{i, j}$ in (4.2a) we obtain that for any $i \in V_{y}, j \in V_{\theta_{+}}$,

$$
\begin{aligned}
q_{i, j} \frac{\mathrm{d} \omega_{i, j}}{\mathrm{~d} t}= & -\frac{V \sin \theta_{j}}{\Delta y}\left(q_{i, j} \omega_{i, j}-q_{i-1, j} \omega_{i-1, j}\right)+\frac{D_{\theta}}{\Delta \theta^{2}}\left(q_{i, j-1} \omega_{i, j-1}+q_{i, j+1} \omega_{i, j+1}-2 q_{i, j} \omega_{i, j}\right) \\
= & -\frac{V \sin \theta_{j}}{\Delta y} q_{i-1, j}\left(\omega_{i, j}-\omega_{i-1, j}\right)+\frac{D_{\theta}}{\Delta \theta^{2}} q_{i, j+1}\left(\omega_{i, j+1}-\omega_{i, j}\right)-\frac{D_{\theta}}{\Delta \theta^{2}} q_{i, j-1}\left(\omega_{i, j}-\omega_{i, j-1}\right) \\
& +\omega_{i, j}\left[-\frac{V \sin \theta_{j}}{\Delta y}\left(q_{i, j}-q_{i-1, j}\right)+\frac{D_{\theta}}{\Delta \theta^{2}}\left(q_{i, j-1}+q_{i, j+1}-2 q_{i, j}\right)\right]
\end{aligned}
$$

By (4.6a), the last term is zero. Rearranging $H^{\prime}\left(\omega_{i, j}\right)(\mathrm{A} .8)$, we deduce 


$$
\begin{aligned}
& q_{i, j} \frac{\mathrm{d} H\left(\omega_{i, j}\right)}{\mathrm{d} t}-\frac{D_{\theta}}{\Delta \theta^{2}}\left[q_{i, j-1} H\left(\omega_{i, j-1}\right)+q_{i, j+1} H\left(\omega_{i, j+1}\right)-2 q_{i, j} H\left(\omega_{i, j}\right)\right] \\
= & -\frac{V \sin \theta_{j}}{\Delta y} q_{i-1, j} H^{\prime}\left(\omega_{i, j}\right)\left(\omega_{i, j}-\omega_{i-1, j}\right)+\frac{2 D_{\theta}}{\Delta \theta^{2}} q_{i, j} H\left(\omega_{i, j}\right) \\
& +\frac{D_{\theta}}{\Delta \theta^{2}} q_{i, j+1}\left[H^{\prime}\left(\omega_{i, j}\right)\left(\omega_{i, j+1}-\omega_{i, j}\right)-H\left(\omega_{i, j+1}\right)\right]-\frac{D_{\theta}}{\Delta \theta^{2}} q_{i, j-1}\left[H^{\prime}\left(\omega_{i, j}\right)\left(\omega_{i, j}-\omega_{i, j-1}\right)+H\left(\omega_{i, j-1}\right)\right] \\
= & -\frac{V \sin \theta_{j}}{\Delta y}\left[q_{i, j} H\left(\omega_{i, j}\right)-q_{i-1, j} H\left(\omega_{i-1, j}\right)\right]-\frac{V \sin \theta_{j}}{\Delta y} q_{i-1, j}\left[H^{\prime}\left(\omega_{i, j}\right)\left(\omega_{i, j}-\omega_{i-1, j}\right)-H\left(\omega_{i, j}\right)+H\left(\omega_{i-1, j}\right)\right] \\
& -\frac{D_{\theta}}{\Delta \theta^{2}} q_{i, j+1}\left[H\left(\omega_{i, j+1}\right)-H\left(\omega_{i, j}\right)-H^{\prime}\left(\omega_{i, j}\right)\left(\omega_{i, j+1}-\omega_{i, j}\right)\right] \\
& -\frac{D_{\theta}}{\Delta \theta^{2}} q_{i, j-1}\left[H^{\prime}\left(\omega_{i, j}\right)\left(\omega_{i, j}-\omega_{i, j-1}\right)-H\left(\omega_{i, j}\right)+H\left(\omega_{i, j-1}\right)\right] \\
& -\frac{V \sin \theta_{j}}{\Delta y}\left(q_{i-1, j}-q_{i, j}\right) H\left(\omega_{i, j}\right)-\frac{D_{\theta}}{\Delta \theta^{2}}\left(q_{i, j-1}+q_{i, j+1}-2 q_{i, j}\right) H\left(\omega_{i, j}\right) .
\end{aligned}
$$

The last two terms offset according to (4.6a), so we deduce that

$$
\begin{aligned}
& q_{i, j} \frac{\mathrm{d} H\left(\omega_{i, j}\right)}{\mathrm{d} t}+\frac{V \sin \theta_{j}}{\Delta y}\left[q_{i, j} H\left(\omega_{i, j}\right)-q_{i-1, j} H\left(\omega_{i-1, j}\right)\right] \\
\leq & \frac{D_{\theta}}{\Delta \theta^{2}}\left[q_{i, j-1} H\left(\omega_{i, j-1}\right)+q_{i, j+1} H\left(\omega_{i, j+1}\right)-2 q_{i, j} H\left(\omega_{i, j}\right)\right],
\end{aligned}
$$

holds for $i \in V_{y}, j \in V_{\theta_{+}}$, where equality holds if and only if $\omega_{i, j}=\omega_{i, j-1}=\omega_{i, j+1}=\omega_{i-1, j}$.

Similarly, by rearranging (4.2a) and employ (4.6b), we have

$$
\begin{aligned}
& q_{i, j} \frac{\mathrm{d} H\left(\omega_{i, j}\right)}{\mathrm{d} t}+\frac{V \sin \theta_{j}}{\Delta y}\left[q_{i+1, j} H\left(\omega_{i+1, j}\right)-q_{i, j} H\left(\omega_{i, j}\right)\right] \\
\leq & \frac{D_{\theta}}{\Delta \theta^{2}}\left[q_{i, j-1} H\left(\omega_{i, j-1}\right)+q_{i, j+1} H\left(\omega_{i, j+1}\right)-2 q_{i, j} H\left(\omega_{i, j}\right)\right],
\end{aligned}
$$

holds for $i \in V_{y}, j \in V_{\theta_{-}}$, where equality holds if and only if $\omega_{i, j}=\omega_{i, j-1}=\omega_{i, j+1}=\omega_{i+1, j}$.

For $i \in V_{y} \backslash\{1,2 I\}, j \in V_{\theta_{0}},(4.2 \mathrm{c})$ and (4.6c) gives

$$
q_{i, j} \frac{\mathrm{d} H\left(\omega_{i, j}\right)}{\mathrm{d} t} \leq \frac{D_{\theta}}{\Delta \theta^{2}}\left[q_{i, j-1} H\left(\omega_{i, j-1}\right)+q_{i, j+1} H\left(\omega_{i, j+1}\right)-2 q_{i, j} H\left(\omega_{i, j}\right)\right],
$$

where equality holds if and only if $\omega_{i, j}=\omega_{i, j-1}=\omega_{i, j+1}$.

Third step: special case where $\frac{\partial p}{\partial \theta}$ jumps. On node $(i, j)=(2 I, J)$, substitute $p_{i, j}$ by $q_{i, j} \omega_{i, j}+q_{i, j}$ in $(4.4$ c) we obtain

$$
\begin{aligned}
q_{2 I, J} \frac{\mathrm{d} \omega_{2 I, J}}{\mathrm{~d} t}= & \frac{D_{\theta}}{\Delta \theta^{2}}\left(q_{2 I, J-1} \omega_{2 I, J-1}+q_{2 I, J+1} \omega_{2 I, J+1}-2 q_{2 I, J} \omega_{2 I, J}+\frac{1}{\Delta y} q_{+, J+1} \omega_{+, J+1}\right) \\
= & \frac{D_{\theta}}{\Delta \theta^{2}}\left[q_{2 I, J-1}\left(\omega_{2 I, J-1}-\omega_{2 I, J}\right)+q_{2 I, J+1}\left(\omega_{2 I, J+1}-\omega_{2 I, J}\right)+\frac{1}{\Delta y} q_{+, J+1}\left(\omega_{+, J+1}-\omega_{2 I, J}\right)\right] \\
& +\frac{D_{\theta}}{\Delta \theta^{2}} \omega_{2 I, J}\left(q_{2 I, J-1}+q_{2 I, J+1}-2 q_{2 I, J}+\frac{1}{\Delta y} q_{+, J+1}\right),
\end{aligned}
$$


in which the last term is zero by (4.7). Multiply both sides with $H^{\prime}\left(\omega_{2 I, J}\right)$, it follows that

$$
\begin{aligned}
& q_{2 I, J} \frac{\mathrm{d} H\left(\omega_{2 I, J}\right)}{\mathrm{d} t}-\frac{D_{\theta}}{\Delta \theta^{2}}\left[q_{2 I, J+1} H\left(\omega_{2 I, J+1}\right)+q_{2 I, J-1} H\left(\omega_{2 I, J-1}\right)-2 q_{2 I, J} H\left(\omega_{2 I, J}\right)\right] \\
= & \frac{D_{\theta}}{\Delta \theta^{2}} q_{2 I, J-1}\left[H^{\prime}\left(\omega_{2 I, J}\right)\left(\omega_{2 I, J-1}-\omega_{2 I, J}\right)-H\left(\omega_{2 I, J-1}\right)\right]+\frac{D_{\theta}}{\Delta \theta^{2}} q_{2 I, J+1}\left[H^{\prime}\left(\omega_{2 I, J}\right)\left(\omega_{2 I, J+1}-\omega_{2 I, J}\right)-H\left(\omega_{2 I, J+1}\right)\right] \\
& +\frac{D_{\theta}}{\Delta \theta^{2} \Delta y} q_{+, J+1} H^{\prime}\left(\omega_{2 I, J}\right)\left(\omega_{+, J+1}-\omega_{2 I, J+1}\right)+\frac{2 D_{\theta}}{\Delta \theta^{2}} q_{2 I, J} H\left(\omega_{2 I, J}\right) \\
= & \frac{D_{\theta}}{\Delta \theta^{2}} q_{2 I, J-1}\left[H^{\prime}\left(\omega_{2 I, J}\right)\left(\omega_{2 I, J-1}-\omega_{2 I, J}\right)-H\left(\omega_{2 I, J-1}\right)+H\left(\omega_{2 I, J}\right)\right] \\
& +\frac{D_{\theta}}{\Delta \theta^{2}} q_{2 I, J+1}\left[H^{\prime}\left(\omega_{2 I, J}\right)\left(\omega_{2 I, J+1}-\omega_{2 I, J}\right)-H\left(\omega_{2 I, J+1}\right)+H\left(\omega_{2 I, J}\right)\right] \\
& +\frac{D_{\theta}}{\Delta \theta^{2} \Delta y} q_{+, J+1}\left[H^{\prime}\left(\omega_{2 I, J}\right)\left(\omega_{+, J+1}-\omega_{2 I, J}\right)-H\left(\omega_{+, J+1}\right)+H\left(\omega_{2 I, J}\right)\right] \\
& +\frac{D_{\theta}}{\Delta \theta^{2} \Delta y} q_{+, J+1} H\left(\omega_{+, J+1}\right)-\frac{D_{\theta}}{\Delta \theta^{2}}\left(q_{2 I, J-1}+q_{2 I, J+1}-2 q_{2 I, J}+\frac{1}{\Delta y} q_{+, J+1}\right) H\left(\omega_{2 I, J}\right) .
\end{aligned}
$$

The last term is zero according to (4.7), so we deduce that

$$
q_{2 I, J} \frac{\mathrm{d} H\left(\omega_{2 I, J}\right)}{\mathrm{d} t} \leq \frac{D_{\theta}}{\Delta \theta^{2}}\left[q_{2 I, J+1} H\left(\omega_{2 I, J+1}\right)+q_{2 I, J-1} H\left(\omega_{2 I, J-1}\right)-2 q_{2 I, J} H\left(\omega_{2 I, J}\right)\right]+\frac{D_{\theta}}{\Delta \theta^{2} \Delta y} q_{+, J+1} H\left(\omega_{+, J+1}\right)
$$

where equality holds if and only if $\omega_{2 I, J-1}=\omega_{2 I, J}=\omega_{2 I, J+1}=\omega_{+, J+1}$.

Similarly, on node $(i, j)=(2 I, 2 J),(1,0),(1, J)$, we have

$$
\begin{aligned}
& q_{2 I, 2 J} \frac{\mathrm{d} H\left(\omega_{2 I, 2 J}\right)}{\mathrm{d} t} \leq \frac{D_{\theta}}{\Delta \theta^{2}}\left[q_{2 I, 2 J+1} H\left(\omega_{2 I, 2 J+1}\right)+q_{2 I, 2 J-1} H\left(\omega_{2 I, 2 J-1}\right)-2 q_{2 I, 2 J} H\left(\omega_{2 I, 2 J}\right)\right] \\
&+\frac{D_{\theta}}{\Delta \theta^{2} \Delta y} q_{+, 2 J-1} H\left(\omega_{+, 2 J-1}\right) \\
& q_{1,0} \frac{\mathrm{d} H\left(\omega_{1,0}\right)}{\mathrm{d} t} \leq \frac{D_{\theta}}{\Delta \theta^{2}}\left[q_{1,1} H\left(\omega_{1,1}\right)+q_{1,-1} H\left(\omega_{1,-1}\right)-2 q_{1,0} H\left(\omega_{1,0}\right)\right]+\frac{D_{\theta}}{\Delta \theta^{2} \Delta y} q_{-, 1} H\left(\omega_{-, 1}\right) \\
& q_{1, J} \frac{\mathrm{d} H\left(\omega_{1, J}\right)}{\mathrm{d} t} \leq \frac{D_{\theta}}{\Delta \theta^{2}}\left[q_{1, J+1} H\left(\omega_{1, J+1}\right)+q_{1, J-1} H\left(\omega_{1, J-1}\right)-2 q_{1, J} H\left(\omega_{1, J}\right)\right]+\frac{D_{\theta}}{\Delta \theta^{2} \Delta y} q_{-, J-1} H\left(\omega_{-, J-1}\right)
\end{aligned}
$$

Fourth step: collection and offsets To summarize, sum up (A.9) for $i \in V_{y}, j \in V_{\theta_{+}}$, (A.10) for $i \in V_{y}, j \in V_{\theta_{-}}$, (A.11) for $i \in V_{y} \backslash\{1,2 I\}, j \in V_{\theta_{0}}$, together with (A.13)-(A.16), the diffusion term offsets and it follows that

$$
\begin{aligned}
& \frac{\mathrm{d}}{\mathrm{d} t} \sum_{i \in V_{y}} \sum_{j \in V_{\theta}} q_{i, j} H\left(\omega_{i, j}\right)+\sum_{j \in V_{\theta_{-}}} \frac{V \sin \theta_{j}}{\Delta y} q_{2 I, j} H\left(\omega_{2 I, j}\right)-\sum_{j \in V_{\theta_{-}}} \frac{V \sin \theta_{j}}{\Delta y} q_{2 I, j} H\left(\omega_{2 I, j}\right) \\
\leq & \frac{D_{\theta}}{\Delta \theta^{2} \Delta y}\left[q_{+, J+1} H\left(\omega_{+, J+1}\right)+q_{+, 2 J-1} H\left(\omega_{+, 2 J-1}\right)+q_{-, 1} H\left(\omega_{-, 1}\right)+q_{-, J-1} H\left(\omega_{+, J-1}\right)\right] .
\end{aligned}
$$

Hence (A.5), (A.7) and (A.17) imply inequality (A.2) in the theorem.

Define

$$
M(t)=\Delta \theta \Delta y \sum_{i \in V_{y}} \sum_{j \in V_{\theta}} q_{i, j} \omega_{i, j}^{2}(t)+\Delta \theta \sum_{j \in V_{\theta_{+}}} q_{+, j} \omega_{+, j}^{2}(t)+\Delta \theta \sum_{j \in V_{\theta_{-}}} q_{-, j} \omega_{-, j}^{2}(t) .
$$


Choose $H(x)=x^{2}$ in (A.2) then we have $\mathrm{d} M(t) / \mathrm{d} t \leq 0$. Suppose the initial data of $\omega_{i, j}$ and $\omega_{ \pm, j}$ satisfies $M(0)<\infty$, then since $M(t)$ decreases monotonically, and it has a lower bound 0 , so $M(t)$ converges as $t$ goes to infinity. While the equality of (A.2) holds if and only if $M(t)=0$, so we have $\lim _{t \rightarrow \infty} M(t)=0$, which implies $\lim _{t \rightarrow \infty} \omega_{i, j}(t)=\lim _{t \rightarrow \infty} \omega_{ \pm, j}(t)=0$. We conclude that $p_{i, j}(t)$ converges to $q_{i, j}$ for any $i \in V_{\theta}, j \in V_{y}$ as $t$ goes to infinity, and $p_{ \pm, j}(t)$ converges to $q_{ \pm, j}$ for any $j \in V_{j, \pm}$ as $t$ goes to infinity.

\section{References}

[1] A. P. Berke, L. Turner, H. C. Berg, and E. Lauga, Hydrodynamic attraction of swimming microorganisms by surfaces, Physical Review Letters, 101 (2008), p. 038102.

[2] L. Berlyand, P.-E. Jabin, M. Potomkin, and E. Ratajczyk, A kinetic approach to active rods dynamics in confined domains, Multiscale Modeling \& Simulation, 18 (2020), pp. 1-20.

[3] M. Bessemoulin-Chatard, M. Herda, And T. Rey, Hypocoercivity and diffusion limit of a finite volume scheme for linear kinetic equations, Math. Comp., 89 (2020), pp. 1093-1133.

[4] S. Bianchi, F. Saglimbeni, and R. Di Leonardo, Holographic imaging reveals the mechanism of wall entrapment in swimming bacteria, Physical Review X, 7 (2017), p. 011010.

[5] M. H. A. Davis, Piecewise-deterministic Markov processes: a general class of nondiffusion stochastic models, J. Roy. Statist. Soc. Ser. B, 46 (1984), pp. 353-388. With discussion.

[6] R. Di Leonardo, L. Angelani, D. Dell'Arciprete, G. Ruocco, V. Iebba, S. Schippa, M. P. Conte, F. Mechrini, F. De Angelis, and E. Di Fabrizio, Bacterial ratchet motors, Proceedings of the National Academy of Sciences, 107 (2010), pp. 9541-9545.

[7] W. R. Diluzio, L. Turner, M. Mayer, P. Garstecki, D. B. Weibel, H. C. Berg, and G. M. Whitesides, Escherichia coli swim on the right-hand side, Nature, 435 (2005), pp. 1271-1274.

[8] J. Dolbeault, C. Mouhot, and C. Schmeiser, Hypocoercivity for linear kinetic equations conserving mass, Trans. Amer. Math. Soc., 367 (2015), pp. 3807-3828.

[9] K. Drescher, J. Dunkel, L. H. Cisneros, S. Ganguly, and R. E. Goldstein, Fluid dynamics and noise in bacterial cell-cell and cell-surface scattering, Proceedings of the National Academy of Sciences, 108 (2011), pp. 10940-10945.

[10] G. Dujardin, F. HÉrau, and P. Lafitte, Coercivity, hypocoercivity, exponential time decay and simulations for discrete Fokker-Planck equations, Numer. Math., 144 (2020), pp. 615-697.

[11] J. Elgeti And G. Gompper, Self-propelled rods near surfaces, EPL (Europhysics Letters), 85 (2009), p. 38002.

[12] B. Ezhilan And D. Saintillan, Transport of a dilute active suspension in pressure-driven channel flow, Journal of Fluid Mechanics, 777 (2015), pp. 482-522.

[13] N. Figueroa-Morales, G. Leonardo Miño, A. Rivera, R. Caballero, E. Clément, E. Altshuler, AND A. Lindner, Living on the edge: transfer and traffic of E. coli in a confined flow, Soft Matter, 11 (2015), pp. 6284-6293.

[14] P. D. Frymier, R. M. Ford, H. C. Berg, and P. T. Cummings, Three-dimensional tracking of motile bacteria near a solid planar surface, Proceedings of the National Academy of Sciences, 92 (1995), pp. 6195-6199. 
[15] P. Galajda, J. Keymer, P. Chaikin, and R. Austin, A wall of funnels concentrates swimming bacteria, Journal of bacteriology, 189 (2007), pp. 8704-8707.

[16] F. HÉRAU, Introduction to hypocoercive methods and applications for simple linear inhomogeneous kinetic models, in Lectures on the analysis of nonlinear partial differential equations. Part 5, vol. 5 of Morningside Lect. Math., Int. Press, Somerville, MA, 2018, pp. 119-147.

[17] P. E. Klofden and E. Platen, Numerical Solution of Stochastic Differential Equations, Springer, Berlin, 1992.

[18] C. F. LEe, Active particles under confinement: aggregation at the wall and gradient formation inside a channel, New Journal of Physics, 15 (2013), p. 055007.

[19] G. Li, J. Bensson, L. Nisimova, D. Munger, P. Mahautmr, J. X. Tang, M. R. Maxey, and Y. V. Brun, Accumulation of swimming bacteibria near a solid surface, Physical Review E, 84 (2011), p. 041932.

[20] G. Li AND J. X. TANG, Accumulation of microswimmers near a surface mediated by collision and rotational brownian motion, Physical review letters, 103 (2009), p. 078101.

[21] P. Michel, S. Mischler, And B. Perthame, General relative entropy inequality: an illustration on growth models, J. Math. Pures Appl., 84 (2005), pp. 1235-1260.

[22] M. Molaei, M. Barry, R. Stocker, and J. Sheng, Failed escape: solid surfaces prevent tumbling of escherichia coli, Physical review letters, 113 (2014), p. 068103.

[23] A. Sokolov, M. M. Apodaca, B. A. Grzybowski, and I. S. Aranson, Swimming bacteria power microscopic gears, Proceedings of the National Academy of Sciences, 107 (2010), pp. 969-974.

[24] S. E. Spagnolie And E. Lauga, Hydrodynamics of self-propulsion near a boundary: predictions and accuracy of far-field approximations, Journal of Fluid Mechanics, 700 (2012), pp. 105-147.

[25] C. Villani, Hypocoercivity, Mem. Amer. Math. Soc., 202 (2009), pp. iv+141.

[26] C. G. Wagner, M. F. Hagan, and A. Baskaran, Steady-state distributions of ideal active brownian particles under confinement and forcing, Journal of Statistical Mechanics: Theory and Experiment, 2017 (2017), p. 043203. 